TRANSACTIONS OF THE

AMERICAN MATHEMATICAL SOCIETY

Volume 359, Number 11, November 2007, Pages 5567-5591

S 0002-9947(07)04270-5

Article electronically published on May 11, 2007

\title{
CONVOLUTIONS AND MULTIPLIER TRANSFORMATIONS OF CONVEX BODIES
}

\author{
FRANZ E. SCHUSTER
}

\begin{abstract}
Rotation intertwining maps from the set of convex bodies in $\mathbb{R}^{n}$ into itself that are continuous linear operators with respect to Minkowski and Blaschke addition are investigated. The main focus is on Blaschke-Minkowski homomorphisms. We show that such maps are represented by a spherical convolution operator. An application of this representation is a complete classification of all even Blaschke-Minkowski homomorphisms which shows that these maps behave in many respects similar to the well known projection body operator. Among further applications is the following result: If an even Blaschke-Minkowski homomorphism maps a convex body to a polytope, then it is a constant multiple of the projection body operator.
\end{abstract}

\section{INTRODUCTION AND STATEMENT OF MAIN RESULTS}

For $n \geq 3$ let $\mathcal{K}^{n}$ be the set of convex bodies in $\mathbb{R}^{n}$, i.e. nonempty, compact, convex sets, equipped with the Hausdorff topology. Let $\mathcal{K}_{0}^{n}$ be the subset of $\mathcal{K}^{n}$ consisting of the convex bodies with interior points. A convex body $K \in \mathcal{K}^{n}$ is determined by its support function $h(K, \cdot)$, defined on $\mathbb{R}^{n}$ by $h(K, x)=\max \{x \cdot y$ : $y \in K\}$. We shall mostly consider the restriction of support functions to the Euclidean unit sphere $S^{n-1}$ which are elements of $\mathcal{C}\left(S^{n-1}\right)$, the space of continuous functions on $S^{n-1}$ with the uniform topology.

By Minkowski's existence theorem, a convex body $K \in \mathcal{K}_{0}^{n}$ is also determined up to translation by its surface area measure (of order $n-1) S_{n-1}(K, \cdot)$. The measure of a Borel set $\omega \subseteq S^{n-1}$ is the $n-1$ dimensional Hausdorff measure of the set of all boundary points of $K$ at which there exists a normal vector of $K$ belonging to $\omega$. $S_{n-1}(K, \cdot)$ is an element of $\mathcal{M}_{o}^{+}\left(S^{n-1}\right)$, the space of nonnegative measures on $S^{n-1}$ having their center of mass in the origin, equipped with the weak ${ }^{*}$ topology.

Two of the most important algebraic structures on the set of convex bodies are Minkowski (vector) addition and Blaschke addition. For $K_{1}, K_{2} \in \mathcal{K}^{n}$ and $\lambda_{1}, \lambda_{2} \geq 0$, the support function of the Minkowski linear combination $\lambda_{1} K_{1}+\lambda_{2} K_{2}$ is

$$
h\left(\lambda_{1} K_{1}+\lambda_{2} K_{2}, \cdot\right)=\lambda_{1} h\left(K_{1}, \cdot\right)+\lambda_{2} h\left(K_{2}, \cdot\right) .
$$

Received by the editors July 4, 2005 and, in revised form, December 7, 2005.

2000 Mathematics Subject Classification. Primary 52A20; Secondary 52A40, 43A90.

Key words and phrases. Convex bodies, Minkowski addition, Blaschke addition, rotation intertwining map, spherical convolution, spherical harmonic, multiplier transformation, projection body, Petty conjecture.

(C)2007 American Mathematical Society Reverts to public domain 28 years from publication 
For $K_{1}, K_{2} \in \mathcal{K}_{0}^{n}$ and $\lambda_{1}, \lambda_{2} \geq 0$ (not both 0 ), the Blaschke linear combination $\lambda_{1} \cdot K_{1} \# \lambda_{2} \cdot K_{2}$ is defined (up to translation) by

$$
S_{n-1}\left(\lambda_{1} \cdot K_{1} \# \lambda_{2} \cdot K_{2}, \cdot\right)=\lambda_{1} S_{n-1}\left(K_{1}, \cdot\right)+\lambda_{2} S_{n-1}\left(K_{2}, \cdot\right) \text {. }
$$

With these operations $\mathcal{K}^{n}$ and $\left[\mathcal{K}_{0}^{n}\right]$, the set of translation classes of convex bodies with nonempty interior are abelian semi-groups.

There are several groups acting on the spaces $\mathcal{K}^{n}$ and $\left[\mathcal{K}_{0}^{n}\right]$. One of the most important is the group of rotations $S O(n)$.

With these different structures on the space of convex bodies it is natural to ask what kind of operators on $\mathcal{K}^{n}$ and $\left[\mathcal{K}_{0}^{n}\right]$ are compatible with the algebraic and topological structures. From a geometric point of view we are interested in operators that intertwine rotations.

In 26] Schneider started an investigation of continuous rigid motion intertwining and Minkowski additive maps which he called Minkowski endomorphisms. Among other results he obtained (under additional assumptions) characterizations of several such mappings. In 27] Schneider classified all Minkowski endomorphisms in $\mathbb{R}^{2}$. Kiderlen, relaxing the definition of Minkowski endomorphisms to continuous rotation intertwining and Minkowski additive maps, extended in [13] Schneider's classification result to higher dimensions for weakly monotone Minkowski endomorphisms, i.e. they are monotone with respect to set inclusion on convex bodies having their Steiner point in the origin. Kiderlen also gave a complete classification of all Blaschke endomorphisms, i.e. continuous rotation intertwining and Blaschke additive maps.

Definition 1.1. A map $\Phi:\left[\mathcal{K}_{0}^{n}\right] \rightarrow \mathcal{K}^{n}$ is called a Blaschke-Minkowski homomorphism if it satisfies the following conditions:

(a) $\Phi$ is continuous.

(b) For all $K, L \in\left[\mathcal{K}_{0}^{n}\right]$,

$$
\Phi(K \# L)=\Phi K+\Phi L .
$$

(c) $\Phi$ is rotation intertwining, i.e. for all $K \in\left[\mathcal{K}_{0}^{n}\right]$ and every $\vartheta \in S O(n)$,

$$
\Phi(\vartheta K)=\vartheta \Phi K
$$

The well known projection body operator, see (1.3), is an example of a BlaschkeMinkowski homomorphism. For its many applications in different areas see [2, [3, [5], 8], 9], [10 and [15]. Further examples of Blaschke-Minkowski homomorphisms can be found in [7] and [12]. The operator that maps every convex body to the origin is called the trivial Blaschke-Minkowski homomorphism.

The main purpose of this article is to show that there is a representation for Blaschke-Minkowski homomorphisms analogous to the ones obtained by Schneider and Kiderlen and to establish a connection to the theory of Minkowski and Blaschke endomorphisms developed by them. Moreover we will characterize special Blaschke-Minkowski homomorphisms and investigate the volume and more general quermassintegrals of images under these mappings.

Classification results of mappings of convex bodies, in particular of valuations on convex sets, form a main part of convex geometry. Here, a map $\Phi$ defined on $\mathcal{K}^{n}$ and taking values in an abelian semigroup is called a valuation if for all $K, L \in \mathcal{K}^{n}$ such that also $K \cup L \in \mathcal{K}^{n}$,

$$
\Phi(K \cup L)+\Phi(K \cap L)=\Phi K+\Phi L .
$$


The theory of valuations and its important applications in integral geometry and geometric probability are developed and described in [11, [14, [21, [22].

In [15] and [16] valuations taking values in $\mathcal{K}^{n}$ are investigated which intertwine volume preserving linear transformations. In Section 4 we will see that BlaschkeMinkowski homomorphisms are special valuations. Thus, our results are a contribution to the classification of continuous rotation intertwining valuations taking values in $\mathcal{K}^{n}$. In full generality this problem is still open.

Let $S O(n)$ denote the group of rotations in $n$ dimensions. Identifying $S^{n-1}$ with the homogeneous space $S O(n) / S O(n-1)$, where $S O(n-1)$ denotes the group of rotations leaving the point $\widehat{e}$ (the pole) of $S^{n-1}$ fixed, it is possible to introduce a natural convolution structure on $\mathcal{C}\left(S^{n-1}\right)$ and $\mathcal{M}\left(S^{n-1}\right)$. Convolution operators generated by $S O(n-1)$ invariant (or zonal) functions and measures play a special role. The set of continuous zonal functions on $S^{n-1}$ will be denoted by $\mathcal{C}\left(S^{n-1}, \widehat{e}\right)$.

A function $f \in \mathcal{C}\left(S^{n-1}\right)$ is called weakly positive if there exists a vector $x \in \mathbb{R}^{n}$ such that $f(u)+x \cdot u \geq 0$ for every $u \in S^{n-1}$. The main theorem of this article is the following representation for Blaschke-Minkowski homomorphisms:

Theorem 1.2. If $\Phi: \mathcal{K}^{n} \rightarrow \mathcal{K}^{n}$ is a Blaschke-Minkowski homomorphism, then there is a weakly positive $g \in \mathcal{C}\left(S^{n-1}, \widehat{e}\right)$, unique up to addition of a linear function, such that

$$
h(\Phi K, \cdot)=S_{n-1}(K, \cdot) * g .
$$

Note that in Theorem 1.2 the domain of $\Phi$ is the set $\mathcal{K}^{n}$ in contrast to Definition 1.1. The reason for this is a natural identification of maps on $\left[\mathcal{K}_{0}^{n}\right]$ with translation invariant maps on $\mathcal{K}_{0}^{n}$ and the fact (as we will show) that there is a unique continuous extension of every Blaschke-Minkowski homomorphism to $\mathcal{K}^{n}$.

A map $\Phi: \mathcal{K}^{n} \rightarrow \mathcal{K}^{n}$ is called even if $\Phi K=\Phi(-K)$ for every $K \in \mathcal{K}^{n}$. We call a body $K \in \mathcal{K}^{n}$ a body of revolution if $K$ is invariant under rotations of $S O(n-1)$. Using Theorem [1.2 and a further investigation of properties of generating functions of Blaschke-Minkowski homomorphisms, a classification of all even Blaschke-Minkowski homomorphisms is possible.

Theorem 1.3. A map $\Phi: \mathcal{K}^{n} \rightarrow \mathcal{K}^{n}$ is an even Blaschke-Minkowski homomorphism if and only if there is a centrally symmetric body of revolution $L \in \mathcal{K}^{n}$, unique up to translation, such that

$$
h(\Phi K, \cdot)=S_{n-1}(K, \cdot) * h(L, \cdot) .
$$

The projection body operator $\Pi: \mathcal{K}^{n} \rightarrow \mathcal{K}^{n}$ is defined by

$$
h(\Pi K, u)=\operatorname{vol}_{n-1}\left(K \mid u^{\perp}\right)=\frac{1}{2}\left(S_{n-1}(K, \cdot) * h([-\widehat{e}, \widehat{e}], \cdot)\right)(u),
$$

where $[-\widehat{e}, \widehat{e}]$ denotes the segment with endpoints $-\widehat{e}$ and $\widehat{e}$. The operator $\Pi$ maps polytopes to finite Minkowski linear combinations of rotated and dilated copies of the line segment $[-\widehat{e}, \widehat{e}]$, which is a geometric interpretation of the convolution formula in (1.3). A general convex body is mapped by $\Pi$ to a zonoid, i.e. a limit of Minkowski sums of line segments. By Theorem [1.3, a general even BlaschkeMinkowski homomorphism maps polytopes to finite Minkowski linear combinations of rotated and dilated copies of a symmetric body of revolution $L$. General convex bodies are again mapped to limits of these finite Minkowski linear combinations. 
In [13] a notion of adjointness between Minkowski and Blaschke endomorphisms was introduced. The following consequence of Theorem 1.2 illustrates the behaviour of adjoint endomorphisms in conjunction with Blaschke-Minkowski homomorphisms.

Theorem 1.4. Let $\Psi$ be a Minkowski and $\Psi^{*}$ a Blaschke endomorphism. Then the following statements are equivalent:

(a) $\Psi$ and $\Psi^{*}$ are adjoint endomorphisms.

(b) For every Blaschke-Minkowski homomorphism $\Phi$

$$
\Phi \circ \Psi^{*}=\Psi \circ \Phi .
$$

(c) (1.4) holds for some injective Blaschke-Minkowski homomorphism $\Phi$.

A different application of Theorem 1.3 is the following characterization of $\Pi$.

Theorem 1.5. Let $\Phi: \mathcal{K}^{n} \rightarrow \mathcal{K}^{n}$ be an even Blaschke-Minkowski homomorphism. If there exists a convex body $K \in \mathcal{K}_{0}^{n}$ such that $\Phi K$ is a polytope, then there is a constant $c \in \mathbb{R}^{+}$such that

$$
\Phi=c \Pi .
$$

As a consequence of Theorem 1.2 the image of a Minkowski linear combination under a Blaschke-Minkowski homomorphism is a homogeneous polynomial of degree $n-1$. In particular, Blaschke-Minkowski homomorphisms satisfy, for $K \in \mathcal{K}^{n}$, the Steiner type formula

$$
\Phi\left(K+\varepsilon B^{n}\right)=\sum_{i=0}^{n-1} \varepsilon^{i}\left(\begin{array}{c}
n-1 \\
i
\end{array}\right) \Phi_{i} K,
$$

where $B^{n}$ is the Euclidean unit ball and the sum is with respect to Minkowski addition. The operators $\Phi_{i}: \mathcal{K}^{n} \rightarrow \mathcal{K}^{n}, i=0, \ldots, n-1$, are continuous and rotation intertwining. The image of a ball under a Blaschke-Minkowski homomorphism $\Phi$ is again a ball. Let in the following $r_{\Phi} \in \mathbb{R}^{+}$denote the radius of $\Phi B^{n}$ and $\kappa_{n}$ the volume of $B^{n}$.

We will prove a strengthened version of the classical inequality between the two consecutive quermassintegrals $W_{n-1}$ and $W_{n-2}$, using the induced weakly monotone Minkowski endomorphisms $\Phi_{n-2}$.

Theorem 1.6. Let $\Phi: \mathcal{K}^{n} \rightarrow \mathcal{K}^{n}$ be a nontrivial Blaschke-Minkowski homomorphism. If $K \in \mathcal{K}^{n}$, then

$$
W_{n-1}(K)^{2} \geq \frac{\kappa_{n}}{r_{\Phi}^{2}} W_{n-2}\left(\Phi_{n-2} K\right) \geq \kappa_{n} W_{n-2}(K) .
$$

If $K$ is not a singleton, there is equality on the left hand side only if $\Phi_{n-2} K$ is a ball and equality on the right hand side only if $K$ is ball.

Inequality (1.5) is related to a conjectured projection inequality of Petty for the volume of projection bodies; see [5], [18] and 24].

\section{SPHERICAl CONVOLUTION AND SPHERICAL HARMONICS}

As we deal with different kinds of analytical representations of convex bodies by functions and measures on $S^{n-1} \cong S O(n) / S O(n-1)$, we will first introduce some basic notions connected to $S O(n)$ and $S^{n-1}$. As a general reference for this section 
we recommend the article by Grinberg and Zhang [9] and the book by Groemer 10 .

The identification of $S^{n-1}$ with $S O(n) / S O(n-1)$ is for $u \in S^{n-1}$ given by

$$
u=\vartheta \widehat{e} \mapsto \vartheta S O(n-1) .
$$

The projection from $S O(n)$ onto $S^{n-1}$ is $\vartheta \mapsto \widehat{\vartheta}:=\vartheta \widehat{e}$. The unity $e \in S O(n)$ is mapped to the pole of the sphere $\widehat{e} \in S^{n-1}$. SO(n) and $S^{n-1}$ will be equipped with the invariant probability measures denoted by $d \vartheta$ and $d u$.

Let $\mathcal{C}(S O(n))$ denote the set of continuous functions on $S O(n)$ with the uniform topology and $\mathcal{M}(S O(n))$ its dual space of signed finite measures on $S O(n)$ with the weak* topology. Let $\mathcal{M}^{+}(S O(n))$ be the set of nonnegative measures on $S O(n)$. For $\mu \in \mathcal{M}(S O(n))$ and $f \in \mathcal{C}(S O(n))$, the canonical pairing is

$$
\langle\mu, f\rangle=\langle f, \mu\rangle=\int_{S O(n)} f(\vartheta) d \mu(\vartheta) .
$$

Sometimes we will identify a continuous function $f$ with the absolute continuous measure with density $f$ and thus view $\mathcal{C}(S O(n))$ as a subspace of $\mathcal{M}(S O(n))$. The canonical pairing is then consistent with the usual inner product on $\mathcal{C}(S O(n))$.

For $\vartheta \in S O(n)$, the left translation $\vartheta f$ of $f \in \mathcal{C}(S O(n))$ is defined by

$$
\vartheta f(\eta)=f\left(\vartheta^{-1} \eta\right)
$$

For $\mu \in \mathcal{M}(S O(n))$, we set

$$
\langle\vartheta \mu, f\rangle=\left\langle\mu, \vartheta^{-1} f\right\rangle
$$

then $\vartheta \mu$ is just the image measure of $\mu$ under the rotation $\vartheta$. For $f \in \mathcal{C}(S O(n))$, the function $\hat{f} \in \mathcal{C}(S O(n))$ is defined by

$$
\hat{f}(\vartheta)=f\left(\vartheta^{-1}\right) .
$$

For a measure $\mu \in \mathcal{M}(S O(n))$, we set

$$
\langle\hat{\mu}, f\rangle=\langle\mu, \hat{f}\rangle .
$$

As $S O(n)$ is a compact Lie group the space $\mathcal{C}(S O(n))$ carries a natural convolution structure. For $f, g \in \mathcal{C}(S O(n))$, the convolution $f * g \in \mathcal{C}(S O(n))$ is defined by

$$
(f * g)(\eta)=\int_{S O(n)} f\left(\eta \vartheta^{-1}\right) g(\vartheta) d \vartheta=\int_{S O(n)} f(\vartheta) g\left(\vartheta^{-1} \eta\right) d \vartheta .
$$

For $\mu \in \mathcal{M}(S O(n))$, the convolutions $\mu * f \in \mathcal{C}(S O(n))$ and $f * \mu \in \mathcal{C}(S O(n))$ with a function $f \in \mathcal{C}(S O(n))$ are defined by

$$
(f * \mu)(\eta)=\int_{S O(n)} f\left(\eta \vartheta^{-1}\right) d \mu(\vartheta), \quad(\mu * f)(\eta)=\int_{S O(n)} \vartheta f(\eta) d \mu(\vartheta) .
$$

Using (2.5), one easily checks that for $\sigma \in \mathcal{M}(S O(n))$ and $f, g \in \mathcal{C}(S O(n))$

$$
\langle g * \sigma, f\rangle=\langle g, f * \hat{\sigma}\rangle \text {. }
$$

This leads to the definition of the convolution of two measures $\mu, \sigma \in \mathcal{M}(S O(n))$

$$
\langle\mu * \sigma, f\rangle=\langle\sigma, \hat{\mu} * f\rangle=\langle\mu, f * \hat{\sigma}\rangle .
$$

The convolution on $\mathcal{M}(S O(n))$ is associative, but as for $n \geq 3$ the group of rotations is not abelian, the convolution on $\mathcal{M}(S O(n))$ is not commutative. For the following Lemma see [9], p.85. 
Lemma 2.1. Let $\mu_{m}, \mu \in \mathcal{M}(S O(n)), m=1,2, \ldots$, and let $f \in \mathcal{C}(S O(n))$. If $\mu_{m} \rightarrow \mu$ weakly, then $f * \mu_{m} \rightarrow f * \mu$ and $\mu_{m} * f \rightarrow \mu * f$ uniformly.

In order to define a convolution structure on $\mathcal{C}\left(S^{n-1}\right)$, we will use the method from Grinberg and Zhang 9 identifying $S^{n-1}$ with $S O(n) / S O(n-1)$. This leads to the identification of $\mathcal{C}\left(S^{n-1}\right)$ with right $S O(n-1)$-invariant functions in $\mathcal{C}(S O(n))$ by

$$
\breve{f}(\vartheta)=f(\vartheta \widehat{e}), \quad f \in \mathcal{C}\left(S^{n-1}\right) .
$$

Conversely, every $f \in \mathcal{C}(S O(n))$ induces a continuous function $\widehat{f}$ on $S^{n-1}$, defined by

$$
\widehat{f}(\widehat{\eta})=\int_{S O(n-1)} f(\eta \vartheta) d \vartheta .
$$

If $f \in \mathcal{C}(S O(n))$ is right $S O(n-1)$ invariant and $g \in \mathcal{C}\left(S^{n-1}\right)$, then $f=\widetilde{\widehat{f}}$ and $g=\widehat{\vec{g}}$. Thus $\mathcal{C}\left(S^{n-1}\right)$ is isomorphic to the subspace of right $S O(n-1)$ invariant functions in $\mathcal{C}(S O(n))$. For a measure $\mu \in \mathcal{M}\left(S^{n-1}\right)$ and a function $f \in \mathcal{C}(S O(n))$, we set

$$
\langle\breve{\mu}, f\rangle=\langle\mu, \widehat{f}\rangle \text {. }
$$

In this way the one-to-one correspondence of functions on $S^{n-1}$ with right $S O(n-1)$ invariant functions on $S O(n)$ carries over to the space $\mathcal{M}\left(S^{n-1}\right)$ and right $S O(n-1)$ invariant measures in $\mathcal{M}(S O(n))$.

Note that definitions (2.1), (2.2) and (2.3), (2.4) now become meaningful for spherical functions and measures. Convolution on $\mathcal{C}\left(S^{n-1}\right)$ can be defined via the identification (2.8). For example the convolution of a function $f \in \mathcal{C}\left(S^{n-1}\right)$ with a measure $\mu \in \mathcal{M}\left(S^{n-1}\right)$ is given by

$$
(f * \mu)(\widehat{\eta})=(\breve{f} * \breve{\mu})(\eta)=\int_{S O(n)} f\left(\eta \vartheta^{-1} \widehat{e}\right) d \breve{\mu}(\vartheta) .
$$

In an analogous way, convolutions of functions or measures can be defined. Note that the Dirac measure $\delta_{\widehat{e}}$ is the unique rightneutral element for the convolution on $S^{n-1}$.

An essential role among spherical functions play $S O(n-1)$ invariant functions. Such a function with the property that $\vartheta f=f$ for every $\vartheta \in S O(n-1)$ is called zonal. Zonal functions depend only on the distance of $u$ to $\widehat{e}$, i.e. on the value $u \cdot \widehat{e}$.

Of course the notion of $S O(n-1)$ invariance carries over to measures as well. We call a measure $\mu \in \mathcal{M}\left(S^{n-1}\right)$ zonal, if $\vartheta \mu=\mu$ for every $\vartheta \in S O(n-1)$. The set of all continuous, zonal functions will be denoted by $\mathcal{C}\left(S^{n-1}, \widehat{e}\right)$, and $\mathcal{M}\left(S^{n-1}, \widehat{e}\right)$ denotes the set of zonal measures on $S^{n-1}$.

Spherical convolution becomes simpler for zonal measures. For $f \in \mathcal{C}\left(S^{n-1}\right)$ and $\mu \in \mathcal{M}\left(S^{n-1}, \widehat{e}\right)$, we have

$$
(f * \mu)(\widehat{\eta})=\langle f, \eta \mu\rangle=\int_{S^{n-1}} f(\eta u) d \mu(u) .
$$

For $f \in \mathcal{C}\left(S^{n-1}\right)$, the rotational symmetrization $\bar{f} \in \mathcal{C}\left(S^{n-1}, \widehat{e}\right)$ is defined by

$$
\bar{f}=\delta_{\widehat{e}} * f=\int_{S O(n-1)} \vartheta f d \vartheta
$$


Since $\delta_{\widehat{e}}$ is the right invariant element for the convolution on $S^{n-1}$, we get

$$
f * g=f * \delta_{\widehat{e}} * g=f * \bar{g} .
$$

Thus, for spherical convolution from the right, it suffices to consider zonal functions and measures. Note that, if $\mu \in \mathcal{M}\left(S^{n-1}, \widehat{e}\right)$, then by (2.9) for every $f \in \mathcal{C}\left(S^{n-1}\right)$

$$
(\vartheta f) * \mu=\vartheta(f * \mu)
$$

for every $\vartheta \in S O(n)$. Thus the spherical convolution from the right is a rotation intertwining operator on $\mathcal{C}\left(S^{n-1}\right)$ and $\mathcal{M}\left(S^{n-1}\right)$.

As a zonal function on $S^{n-1}$ depends only on the value of $u \cdot \widehat{e}$, there is a natural isomorphism between functions and measures on $[-1,1]$ and zonal functions and measures on $S^{n-1}$. Define a map $\Lambda: \mathcal{C}\left(S^{n-1}, \widehat{e}\right) \rightarrow \mathcal{C}([-1,1]), f \mapsto \Lambda f$, by

$$
\Lambda f(t)=f\left(t \widehat{e}+\sqrt{1-t^{2}} v\right), \quad v \in \widehat{e}^{\perp} \cap S^{n-1} .
$$

Then it is easy to see that $\Lambda$ is an isomorphism with inverse

$$
\Lambda^{-1}: \mathcal{C}([-1,1]) \rightarrow \mathcal{C}\left(S^{n-1}, \widehat{e}\right), f \mapsto f(\widehat{e} \cdot .) .
$$

For a zonal measure $\mu \in \mathcal{M}\left(S^{n-1}, \widehat{e}\right)$ and a function $f \in \mathcal{C}([-1,1])$, define

$$
\langle\Lambda \mu, f\rangle=\left\langle\mu, \Lambda^{-1} f\right\rangle \text {. }
$$

The map $\Lambda: \mathcal{M}\left(S^{n-1}, \widehat{e}\right) \rightarrow \mathcal{M}([-1,1])$ is the extension of the map defined in (2.12), and it is again an isomorphism between $\mathcal{M}\left(S^{n-1}, \widehat{e}\right)$ and $\mathcal{M}([-1,1])$ with inverse

$$
\left\langle\Lambda^{-1} \mu, f\right\rangle=\langle\mu, \Lambda \bar{f}\rangle, \quad \mu \in \mathcal{M}([-1,1]), f \in \mathcal{C}\left(S^{n-1}\right) .
$$

The isomorphism $\Lambda$ allows one to identify the dual space of $\mathcal{C}\left(S^{n-1}, \widehat{e}\right)$ with the space $\mathcal{M}\left(S^{n-1}, \widehat{e}\right)$. Using this identification, we obtain for $\mu, \nu \in \mathcal{M}\left(S^{n-1}, \widehat{e}\right)$ and $f \in \mathcal{C}\left(S^{n-1}, \widehat{e}\right)$,

$$
\langle\mu * \nu, f\rangle=\int_{S^{n-1}} \int_{S^{n-1}} \Lambda f(u \cdot v) d \mu(u) d \nu(v)=\langle\nu * \mu, f\rangle .
$$

Thus, the convolution of zonal functions and measures is abelian and $\mathcal{M}\left(S^{n-1}, \widehat{e}\right)$ with the convolution structure becomes an abelian Banach algebra.

Another property of zonal measures $\mu \in \mathcal{M}\left(S^{n-1}, \widehat{e}\right)$ is

$$
\hat{\mu}=\mu \text {. }
$$

As a consequence of (2.6) and (2.14) we obtain the following important lemma.

Lemma 2.2. Let $\mu, \nu \in \mathcal{M}\left(S^{n-1}\right)$ and $f \in \mathcal{C}\left(S^{n-1}\right)$; then

$$
\langle\mu * \nu, f\rangle=\langle\mu, f * \nu\rangle \text {. }
$$

Using Lemma 2.2 and (2.13), we get for $\mu \in \mathcal{M}\left(S^{n-1}\right)$ and $f \in \mathcal{C}\left(S^{n-1}, \widehat{e}\right)$,

$$
(\mu * f)(u)=\int_{S^{n-1}} \Lambda f(u \cdot v) d \mu(v) .
$$

We will frequently use zonal approximate identities $\left(\varphi_{k}\right)_{k \in \mathbb{N}}$. These are nonnegative functions in $\mathcal{C}^{\infty}\left(S^{n-1}\right)$. They have already been considered by Berg 1, and we just briefly recall their most important properties in the following lemma.

Lemma 2.3. Let $\left(\varphi_{k}\right)_{k \in \mathbb{N}}$ be a zonal approximate identity. Then

(a) $f * \varphi_{k} \in \mathcal{C}^{\infty}\left(S^{n-1}\right)$ and $\lim _{k \rightarrow \infty} f * \varphi_{k}=f$ uniformly for every $f \in \mathcal{C}\left(S^{n-1}\right)$.

(b) $\mu * \varphi_{k} \in \mathcal{C}^{\infty}\left(S^{n-1}\right)$ and $\lim _{k \rightarrow \infty} \mu * \varphi_{k}=\mu$ weakly for every $\mu \in \mathcal{M}\left(S^{n-1}\right)$. 
We now collect some facts from the theory of spherical harmonics. A spherical harmonic of dimension $n$ and order $k$ is the restriction to $S^{n-1}$ of a harmonic polynomial of order $k$ in $n$ variables. Let $\mathcal{H}_{k}^{n}$ denote the space of spherical harmonics of dimension $n$ and order $k$. $\mathcal{H}^{n}$ will denote the space of all finite sums of spherical harmonics of dimension $n$.

$\mathcal{H}_{k}^{n}$ is a finite dimensional vector space of dimension $N(n, k)$. The spaces $\mathcal{H}_{k}^{n}$ are pairwise orthogonal with respect to the usual inner product on $\mathcal{C}\left(S^{n-1}\right)$. By definition, $\mathcal{H}_{k}^{n}$ is invariant with respect to rotations. Moreover, $\mathcal{H}_{k}^{n}$ is irreducible, i.e. $\{0\}$ and $\mathcal{H}_{k}^{n}$ are the only subspaces invariant under $S O(n)$. As a consequence we have the following version of Schur's Lemma for spherical harmonics.

Lemma 2.4. Let $\Phi: \mathcal{H}_{k}^{n} \rightarrow \mathcal{M}\left(S^{n-1}\right)$ be a linear map that intertwines rotations. Then $\Phi$ is either injective or the zero map.

If $H_{1}, \ldots, H_{N(n, k)}$ is an orthonormal basis of $\mathcal{H}_{k}^{n}$, then there is a unique polynomial $P_{k}^{n} \in \mathcal{C}([-1,1])$ of degree $k$ such that

$$
\sum_{i=1}^{N(n, k)} H_{i}(u) H_{i}(v)=N(n, k) P_{k}^{n}(u \cdot v) .
$$

The polynomial $P_{k}^{n}$ is called the Legendre polynomial of dimension $n$ and order $k$. The zonal function $u \mapsto P_{k}^{n}(\widehat{e} \cdot u)$ is up to a multiplicative constant the unique zonal spherical harmonic in $\mathcal{H}_{k}^{n}$.

The collection $\left\{H_{1}, \ldots, H_{N(n, k)}: k \in \mathbb{N}\right\}$ forms a complete orthogonal system in $\mathcal{L}^{2}\left(S^{n-1}\right)$, i.e. for every square integrable function $f$ the series

$$
f \sim \sum_{k=0}^{\infty} \pi_{k} f
$$

converges in quadratic mean to $f$, where $\pi_{k} f \in \mathcal{H}_{k}^{n}$ is the orthogonal projection of $f$ on the space $\mathcal{H}_{k}^{n}$. Using (2.16) and (2.9), we obtain

$$
\pi_{k} f=\sum_{i=1}^{N(n, k)}\left\langle f, H_{i}\right\rangle H_{i}=N(n, k)\left(f * P_{k}^{n}(\widehat{e} \cdot .)\right) .
$$

This leads to the definition of the spherical expansion of a measure $\mu \in \mathcal{M}\left(S^{n-1}\right)$

$$
\mu \sim \sum_{k=0}^{\infty} \pi_{k} \mu
$$

where $\pi_{k} \mu \in \mathcal{H}_{k}^{n}$ is defined by

$$
\pi_{k} \mu=N(n, k)\left(\mu * P_{k}^{n}(\widehat{e} \cdot .)\right) .
$$

We note here two special cases of (2.19),

$$
\pi_{0} \mu=\mu * 1 \quad \text { and } \quad \pi_{1} \mu=n \mu *(\widehat{e} \cdot .) .
$$

By Lemma 2.2, we have for every $f \in \mathcal{C}\left(S^{n-1}\right)$

$$
\left\langle\pi_{k} \mu, f\right\rangle=N(n, k)\left\langle\mu * P_{k}^{n}(\widehat{e} \cdot .), f\right\rangle=N(n, k)\left\langle\mu, f * P_{k}^{n}(\widehat{e} \cdot .)\right\rangle=\left\langle\mu, \pi_{k} f\right\rangle,
$$

which, by the completeness of the system of spherical harmonics, immediately gives:

Lemma 2.5. Let $\mu \in \mathcal{M}\left(S^{n-1}\right)$. If $\mu * P_{k}^{n}(\widehat{e} \cdot)=$.0 for every $k \in \mathbb{N}$, then $\mu=0$. 
By Lemma 2.5. $\mu \in \mathcal{M}\left(S^{n-1}\right)$ is uniquely determined by its series expansion (2.18). Zonal functions and measures are even determined by a sequence of real numbers. To see this, note that

$$
\delta_{\widehat{e}} * P_{k}^{n}(u \cdot .)=P_{k}^{n}(\widehat{e} \cdot u) P_{k}^{n}(\widehat{e} \cdot .),
$$

and thus, by (2.15) and (2.7),

$\left(\mu * P_{k}^{n}(\widehat{e} \cdot).\right)(u)=\left\langle\mu, P_{k}^{n}(u \cdot).\right\rangle=\left\langle\mu, \delta_{\widehat{e}} * P_{k}^{n}(u \cdot).\right\rangle=\left\langle\mu, P_{k}^{n}(\widehat{e} \cdot).\right\rangle P_{k}^{n}(\widehat{e} \cdot u)$.

Hence the series expansion of a zonal measure $\mu$ becomes

$$
\mu \sim \sum_{k=0}^{\infty} N(n, k)\left\langle\mu, P_{k}^{n}(\widehat{e} \cdot .)\right\rangle P_{k}^{n}(\widehat{e} \cdot .) .
$$

The numbers $\mu_{k}:=\left\langle\mu, P_{k}^{n}(\widehat{e} \cdot)\right\rangle$ are called Legendre coefficients of $\mu \in \mathcal{M}\left(S^{n-1}, \widehat{e}\right)$. Using $\pi_{k} H=H$ for every $H \in \mathcal{H}_{k}^{n}$ and the fact that spherical convolution of zonal measures is commutative, we obtain a version of the Funk-Hecke Theorem.

Corollary 2.6. If $\mu \in \mathcal{M}\left(S^{n-1}, \widehat{e}\right)$ and $H \in \mathcal{H}_{k}^{n}$, then $H * \mu=\mu_{k} H$.

We are now ready to give the definition of multiplier operators.

Definition 2.7. We call a map $\Phi: \mathcal{Q} \subseteq \mathcal{M}\left(S^{n-1}\right) \rightarrow \mathcal{M}\left(S^{n-1}\right)$ a multiplier transformation if there is a sequence of real numbers $c_{k}$ such that, for every $k \in \mathbb{N}$,

$$
\pi_{k} \Phi \mu=c_{k} \pi_{k} \mu, \quad \forall \mu \in \mathcal{Q} .
$$

The numbers $c_{0}, c_{1}, c_{2}, \ldots$ are called the multipliers of $\Phi$.

Again using the fact that spherical convolution of zonal measures is commutative, we see that for $\mu \in \mathcal{M}\left(S^{n-1}, \widehat{e}\right)$ the map $\Phi_{\mu}: \mathcal{M}\left(S^{n-1}\right) \rightarrow \mathcal{M}\left(S^{n-1}\right)$

$$
\nu \mapsto \nu * \mu
$$

is a multiplier transformation. The sequence of multipliers of these convolution operators is just the sequence of Legendre coefficients of the measure $\mu$.

By definition (2.19) of the orthogonal projection $\pi_{k}$ and (2.11), it is easy to see that multiplier transformations intertwine rotations and that, by definition (2.21), they are linear on the space $\mathcal{H}^{n}$. The following corollary to Schur's Lemma establishes the converse statement; see [26], p.67.

Theorem 2.8. If $\Phi: \mathcal{H}^{n} \rightarrow \mathcal{M}\left(S^{n-1}\right)$ is an intertwining linear map, then $\Phi$ is a multiplier transformation.

\section{CONVEX BOdies AND MUltiplier TRANSFORMATIONS}

Here we collect further material on convex geometry and endomorphisms of convex bodies; see [28, 26] and [13. We also prove that every Blaschke-Minkowski homomorphism is a multiplier transformation.

The volume of a Minkowski linear combination $\lambda_{1} K_{1}+\ldots+\lambda_{m} K_{m}$ of convex bodies $K_{1}, \ldots, K_{m}$ is a homogeneous polynomial of degree $n$ in the $\lambda_{i}$,

$$
V\left(\lambda_{1} K_{1}+\ldots+\lambda_{m} K_{m}\right)=\sum_{i_{1}, \ldots, i_{n}} V\left(K_{i_{1}}, \ldots, K_{i_{n}}\right) \lambda_{i_{1}} \cdots \lambda_{i_{n}} .
$$

The coefficients $V\left(K_{i_{1}}, \ldots, K_{i_{n}}\right)$ are called mixed volumes of $K_{i_{1}}, \ldots, K_{i_{n}}$. These functionals are nonnegative, translation invariant, monotone (with respect to set 
inclusion) and multilinear with respect to Minkowski addition. Denote by $V_{i}(K, L)$ the mixed volume $V(K, \ldots, K, L, \ldots, L)$, where $K$ appears $n-i$ times and $L$ appears $i$ times. The quermassintegrals $W_{i}(K)$ are given by $V_{i}\left(K, B^{n}\right)$.

Let $\mathcal{K}_{i}^{n}$ be the subset of $\mathcal{K}^{n}$ consisting of convex bodies whose dimension is at least $n-i$. Then $K \in \mathcal{K}_{i}^{n}$ if and only if $W_{i}(K)>0$. The classical inequality between two consecutive quermassintegrals states that for $K \in \mathcal{K}^{n}$ and $0 \leq i \leq n-2$,

$$
W_{i+1}(K)^{n-i} \geq \kappa_{n} W_{i}(K)^{n-i-1},
$$

where $\kappa_{n}$ is the volume of the Euclidean unit ball $B^{n}$. If $K \in \mathcal{K}_{i+1}^{n}$ there is equality in (3.1) if and only if $K$ is a ball.

For the functional $V_{1}(K, L)$ there is an integral representation

$$
V_{1}(K, L)=\frac{1}{n}\left\langle h(L, \cdot), S_{n-1}(K, \cdot)\right\rangle .
$$

This shows that $V_{1}:\left[\mathcal{K}_{0}^{n}\right] \times \mathcal{K}^{n} \rightarrow \mathbb{R}$ is bilinear with respect to Blaschke and Minkowski addition.

Also the surface area measure of a Minkowski linear combination of convex bodies $K_{1}, \ldots, K_{m}$ can be expressed as a polynomial homogeneous of degree $n-1$ :

$$
S_{n-1}\left(\lambda_{1} K_{1}+\ldots+\lambda_{m} K_{m}, \cdot\right)=\sum_{i_{1}, \ldots, i_{n-1}} \lambda_{i_{1}} \cdots \lambda_{i_{n-1}} S\left(K_{i_{1}}, \ldots, K_{i_{n-1}}, \cdot\right) .
$$

The coefficients $S\left(K_{i_{1}}, \ldots, K_{i_{n-1}}, \cdot\right) \in \mathcal{M}_{o}^{+}\left(S^{n-1}\right)$ are called the mixed surface area measures of $K_{i_{1}}, \ldots, K_{i_{n}}$. They have the property that for any convex body $K$

$$
V\left(K, K_{1}, \ldots, K_{n-1}\right)=\frac{1}{n}\left\langle h(K, \cdot), S\left(K_{1}, \ldots, K_{n-1}, \cdot\right)\right\rangle .
$$

The measures $S_{j}(K, \cdot):=S\left(K, \ldots, K, B^{n}, \ldots, B^{n}, \cdot\right)$, where $K$ appears $j$ times and $B^{n}$ appears $n-1-j$ times, are called the area measures of order $j$ of $K$.

By (2.20) and (3.4), we have for $K \in \mathcal{K}^{n}$,

$$
W_{n-1}(K)=\kappa_{n} \pi_{0} h(K, \cdot) \quad \text { and } \quad W_{1}(K)=\frac{1}{n} \pi_{0} S_{n-1}(K, \cdot) .
$$

A convex body $K \in \mathcal{K}^{n}$ is uniquely determined by its support function $h(K, \cdot)$, which is positively homogeneous of degree one and sublinear. Conversely, every function with these properties is the support function of a convex body. By (2.1), we have $\vartheta h(K, \cdot)=h(\vartheta K, \cdot)$ for $\vartheta \in S O(n)$. Thus the support function of a convex body $K$ is zonal if and only if $K$ is a body of revolution.

The Steiner point map $s: \mathcal{K}^{n} \rightarrow \mathbb{R}^{n}$, defined by

$$
s(K)=n \int_{S^{n-1}} h(K, u) u d u,
$$

is up to a multiplicative constant the unique vector valued continuous, rotation intertwining and Minkowski additive map; see [26]. Since vector addition in $\mathbb{R}^{n}$ coincides with Minkowski addition of singletons, it is possible to give an alternative definition of the Steiner point

$$
h(\{s(K)\}, \cdot)=n h(K, \cdot) *(\widehat{e} \cdot .)=\pi_{1} h(K, \cdot) .
$$

A convex body $K \in \mathcal{K}_{0}^{n}$ is also uniquely determined up to translation by its surface area measure $S_{n-1}(K, \cdot)$ which is an element of $\mathcal{M}_{o}^{+}\left(S^{n-1}\right)$, the set of nonnegative measures on the sphere with center of mass in the origin. Conversely, every element of $\mathcal{M}_{o}^{+}\left(S^{n-1}\right)$ that is not concentrated on any great sphere is the surface area measure of a convex body with interior points. For $\vartheta \in S O(n)$, we 
have $\vartheta S_{n-1}(K, \cdot)=S_{n-1}(\vartheta K, \cdot)$ and again the surface area measure of a convex body $K$ is zonal if and only if $K$ is a body of revolution.

There is no nonzero vector valued map from the set of translation classes of convex bodies $\left[\mathcal{K}_{0}^{n}\right]=\mathcal{K}_{0}^{n} / \mathbb{R}^{n}$ that is continuous, rotation intertwining and additive with respect to Blaschke addition. This fact is reflected by the relation

$$
\pi_{1} S_{n-1}(K, \cdot)=n S_{n-1}(K, \cdot) *(\widehat{e} \cdot .)=0 .
$$

By (3.6), the Steiner point map can be interpreted as a convolution operator on the set of convex bodies. We will consider in the following more general transformations induced by convolution operators. By (2.5), the convolution from the left with measures $\mu \in \mathcal{M}^{+}(S O(n))$ can be interpreted as (weighted) rotation means. The following consequences of this interpretation appear in $[9]$.

Lemma 3.1. Let $\mu \in \mathcal{M}^{+}(S O(n))$.

(a) For $K \in \mathcal{K}^{n}$, the function $\mu * h(K, \cdot)$ is the support function of a convex body.

(b) For $L \in \mathcal{K}_{0}^{n}$ and $\mu \neq 0$, the measure $\mu * S_{n-1}(L, \cdot)$ is the surface area measure of a convex body with interior points.

By (2.10) and the remarks after Definition 2.7. spherical convolution operators from the right are multiplier transformations. It follows from (3.7) that the convolution of surface area measures with a nonnegative zonal measure $\mu$ again gives nonnegative measures with center of mass in the origin. It is also not hard to see that $S_{n-1}(K, \cdot) * \mu$ is not concentrated on any great sphere. Thus, the measure $S_{n-1}(K, \cdot) * \mu$ is again a surface area measure of a convex body. Noting (3.7), we see that it is sufficient that the measure $\mu$ is positive up to addition of a measure with density $c(\widehat{e} \cdot$.$) . We capture this property of a measure in the following definition:$

Definition 3.2. A measure $\mu \in \mathcal{M}\left(S^{n-1}, \widehat{e}\right)$ is called weakly positive if it is nonnegative up to addition of a linear measure, i.e. a measure with density $c(\widehat{e} \cdot$.$) ,$ $c \in \mathbb{R}$.

It was shown in [13] that also the cone of support functions is invariant under convolution of zonal weakly positive measures. We summarize these results in

Lemma 3.3. Let $\mu \in \mathcal{M}\left(S^{n-1}, \widehat{e}\right)$ be weakly positive.

(a) For $K \in \mathcal{K}^{n}$ the function $h(K, \cdot) * \mu$ is the support function of a convex body.

(b) For $L \in \mathcal{K}_{0}^{n}$ and $\mu$ not linear the measure $S_{n-1}(L, \cdot) * \mu$ is the surface area measure of a convex body with interior points.

We call a map $\Phi: \mathcal{K}^{n} \rightarrow \mathcal{K}^{n}$ that is continuous, rotation intertwining and Minkowski additive a Minkowski endomorphism. A Blaschke endomorphism is a map $\Psi:\left[\mathcal{K}_{0}^{n}\right] \rightarrow\left[\mathcal{K}_{0}^{n}\right]$ that is continuous, rotation intertwining and additive with respect to Blaschke addition.

Let $K, L \in \mathcal{K}^{n}$. Then $K \subseteq L$ if and only if $h(K, \cdot) \leq h(L, \cdot)$. Thus a map $\Phi: \mathcal{K}^{n} \rightarrow \mathcal{K}^{n}$ defined by

$$
h(\Phi K, \cdot)=h(K, \cdot) * \mu,
$$

with a weakly positive measure $\mu \in \mathcal{M}\left(S^{n-1}, \widehat{e}\right)$ is, by (3.6), monotone (with respect to set inclusion) on the set of convex bodies having their Steiner point in the origin. We call a Minkowski endomorphism with this property weakly monotone. 
A classification of weakly monotone Minkowski endomorphisms and Blaschke endomorphisms was established by Kiderlen in 13. We summarize his results in

Theorem 3.4. A map $\Phi: \mathcal{K}^{n} \rightarrow \mathcal{K}^{n}$ is a weakly monotone Minkowski endomorphism if and only if there is a unique weakly positive measure $\mu \in \mathcal{M}\left(S^{n-1}, \widehat{e}\right)$ such that

$$
h(\Phi K, \cdot)=h(K, \cdot) * \mu, \quad K \in \mathcal{K}^{n} .
$$

A map $\Psi:\left[\mathcal{K}_{0}^{n}\right] \rightarrow\left[\mathcal{K}_{0}^{n}\right]$ is a Blaschke endomorphism if and only if there is a weakly positive measure $\nu \in \mathcal{M}\left(S^{n-1}, \widehat{e}\right)$, unique up to addition of a linear measure, such that

$$
S_{n-1}(\Psi K, \cdot)=S_{n-1}(K, \cdot) * \nu, \quad K \in \mathcal{K}_{0}^{n} .
$$

The major open problem concerning Minkowski endomorphisms is a classification without the extra assumption of weak monotonicity. For $n=2$, Schneider obtained in 27. such a result by showing that every Minkowski endomorphism is weakly monotone. The following conjecture appears implicitly in [27] and [13.

Conjecture 3.5. For $n \geq 3$ every Minkowski endomorphism is weakly monotone.

In [13] a natural notion of adjointness between Minkowski and Blaschke endomorphisms was introduced.

Definition 3.6. A Minkowski endomorphism $\Phi$ and a Blaschke endomorphism $\Psi$ are called adjoint if for every $K \in \mathcal{K}_{0}^{n}$ and every $L \in \mathcal{K}^{n}$

$$
V_{1}(\Psi K, L)=V_{1}(K, \Phi L) .
$$

Using (3.2), Lemma2.2 and Theorem 3.4 we see that a Blaschke and a Minkowski endomorphism are adjoint if and only if they have the same generating measure up to addition of a linear measure. By Theorem 3.4 every Blaschke endomorphism has an adjoint weakly monotone Minkowski endomorphism. The converse statement is equivalent to Conjecture 3.5 .

The results obtained in Theorem 3.4 show that the respective endomorphisms are multiplier transformations. This fact has been deduced for Minkowski endomorphisms in [26] using a different method. In the following we will adapt the technique by Schneider to show that also Blaschke-Minkowski homomorphisms induce multiplier transformations.

Every Blaschke-Minkowski homomorphism $\Phi:\left[\mathcal{K}_{0}^{n}\right] \rightarrow \mathcal{K}^{n}$ induces a map on the set of surface area measures by

$$
\Phi S_{n-1}(K, \cdot)=h(\Phi K, \cdot), \quad K \in \mathcal{K}_{0}^{n} .
$$

Using Theorem 2.8, we obtain:

Theorem 3.7. Let $\Phi:\left[\mathcal{K}_{0}^{n}\right] \rightarrow \mathcal{K}^{n}$ be a Blaschke-Minkowski homomorphism. Then the induced map on the set of surface area measures is a multiplier transformation, i.e. there is a sequence $c_{k} \in \mathbb{R}$ such that, for every $K \in \mathcal{K}_{0}^{n}$,

$$
\pi_{k} h(\Phi K, \cdot)=\pi_{k} \Phi S_{n-1}(K, \cdot)=c_{k} \pi_{k} S_{n-1}(K, \cdot) .
$$

For the proof of Theorem 3.7 we need some well known facts on the vector space of differences of surface area measures; see [33] and [10], p.70. 
Lemma 3.8. Let $\mathcal{Q} \subseteq \mathcal{M}_{o}^{+}\left(S^{n-1}\right)$ denote the set of surface area measures of convex bodies with interior points. Then

(a) $\mathcal{Q}$ is dense in $\mathcal{M}_{o}^{+}\left(S^{n-1}\right)$ and $\mathcal{M}_{o}\left(S^{n-1}\right)=\mathcal{Q}-\mathcal{Q}$.

(b) $\mathcal{Q} \cap \mathcal{H}^{n}$ is dense in $\mathcal{Q}$.

Proof of Theorem 3.7. By the additivity property of Blaschke-Minkowski homomorphisms, the induced map (3.10) on the cone $\mathcal{Q}$ of surface area measures of convex bodies is linear, and hence by Lemma 3.8 (a), there is a unique linear extension $\tilde{\Phi}$ to the vector space $\mathcal{M}\left(S^{n-1}\right)$ given by

$$
\tilde{\Phi}(\mu)=\Phi S_{n-1}\left(K_{+}, \cdot\right)-\Phi S_{n-1}\left(K_{-}, \cdot\right)
$$

where $\mu-\pi_{1} \mu=S_{n-1}\left(K_{+}, \cdot\right)-S_{n-1}\left(K_{-}, \cdot\right) \in \mathcal{M}_{o}\left(S^{n-1}\right)$ for some $K_{+}, K_{-} \in \mathcal{K}_{0}^{n}$.

The restriction of $\tilde{\Phi}$ to $\mathcal{H}^{n}$ is by definition linear and intertwines rotations. Thus, by Theorem 2.8, it is a multiplier transformation. The result follows since $\tilde{\Phi}$ and $\Phi$ coincide on the set $\mathcal{Q} \cap \mathcal{H}^{n}$ which is dense in $\mathcal{Q}$ by Lemma 3.8 (b).

By Cauchy's surface area formula, the mean width of the projection body of a convex body $K \in \mathcal{K}_{0}^{n}$ is a constant multiple of the surface area of $K$. The following corollary to Theorem 3.7 is a generalization of this fact.

Corollary 3.9. Let $\Phi:\left[\mathcal{K}_{0}^{n}\right] \rightarrow \mathcal{K}^{n}$ be a Blaschke-Minkowski homomorphism. Then

$$
W_{n-1}(\Phi K)=r_{\Phi} W_{1}(K),
$$

where $r_{\Phi} \in \mathbb{R}^{+}$is the radius of the ball $\Phi B^{n}$.

Proof. We first show that $\Phi B^{n}$ is a ball. To see this, note that $\pi_{k} S_{n-1}\left(B^{n}, \cdot\right)=0$ for $k \geq 1$. Thus by Theorem 3.7, we have $\pi_{k} h\left(\Phi B^{n}, \cdot\right)=0$ for $k \geq 1$, hence $\Phi B^{n}$ is a ball. By Theorem 3.7, the radius $r_{\Phi}$ of $\Phi B^{n}$ is given by

$$
r_{\Phi}=\pi_{0} h\left(\Phi B^{n}, \cdot\right)=\pi_{0} \Phi S_{n-1}\left(B^{n}, \cdot\right)=c_{0} \pi_{0} S_{n-1}\left(B^{n}, \cdot\right)=c_{0} \omega_{n},
$$

where $c_{0}$ denotes the first multiplier of $\Phi$ and $\omega_{n}$ is the surface area of $B^{n}$. By (3.5), we have $W_{n-1}(\Phi K)=\kappa_{n} \pi_{0} h(\Phi K)$ and thus, again by Theorem 3.7 and (3.5),

$$
W_{n-1}(\Phi K)=\kappa_{n} \pi_{0} \Phi S_{n-1}(K, \cdot)=\frac{r_{\Phi}}{n} \pi_{0} S_{n-1}(K, \cdot)=r_{\Phi} W_{1}(K) .
$$

\section{Characterization of Blaschke-Minkowski homomorphisms}

We now turn to the proofs of the main theorems. From now on we will view a map $\Phi:\left[\mathcal{K}_{0}^{n}\right] \rightarrow \mathcal{K}^{n}$ via the obvious identification as a translation invariant map on $\mathcal{K}_{0}^{n}$. The next lemma shows that every Blaschke-Minkowski homomorphism has a unique continuous extension to $\mathcal{K}^{n}$.

Lemma 4.1. Let $\Phi: \mathcal{K}_{0}^{n} \rightarrow \mathcal{K}^{n}$ be a Blaschke-Minkowski homomorphism. Then there is a unique continuous extension of $\Phi$ to $\mathcal{K}^{n}$.

Proof. Let $K_{m} \in \mathcal{K}_{0}^{n}$ be a sequence converging to $K \in \mathcal{K}^{n}$. Then we define

$$
\Phi K=\lim _{m \rightarrow \infty} \Phi K_{m}
$$

To see that this limit exists, note that, by Corollary 3.9, $W_{n-1}\left(\Phi K_{m}\right)=r_{\Phi} W_{1}\left(K_{m}\right)$. Thus, $W_{n-1}\left(\Phi K_{m}\right) \rightarrow r_{\Phi} W_{1}(K)$ as $m \rightarrow \infty$. Hence the sequence $\Phi K_{m}$ is bounded. Let $\Phi K_{m_{j}}$ be a convergent subsequence of $\Phi K_{m}$ with limit $L \in \mathcal{K}^{n}$. By Theorem 3.7 and (2.19),

$$
\pi_{k} h\left(\Phi K_{m_{j}}, \cdot\right)=c_{k} \pi_{k} S_{n-1}\left(K_{m_{j}}, \cdot\right)=c_{k} N(n, k) S_{n-1}\left(K_{m_{j}}, \cdot\right) * P_{k}^{n}(\widehat{e} \cdot .) .
$$


By Lemma 2.1, this converges uniformly to $c_{k} \pi_{k} S_{n-1}(K, \cdot)$. On the other hand, $\pi_{k} h\left(\Phi K_{m_{j}}, \cdot\right) \rightarrow \pi_{k} h(L, \cdot)$ as $j \rightarrow \infty$. By the completeness of spherical harmonics, this implies that the limit of every convergent subsequence $\Phi K_{m_{j}}$ of $\Phi K_{m}$ coincides and thus $\Phi K_{m}$ itself is convergent.

We will need a criterion to determine if a measure $\mu \in \mathcal{M}\left(S^{n-1}, \widehat{e}\right)$ is weakly positive. Let $\mathcal{L}=\left\{h(K, \cdot)-h(L, \cdot): K, L \in \mathcal{K}^{n}\right\}$ denote the vector space of differences of support functions. The following lemma is in a slightly weaker form due to Schneider [27] for $n=2$ and Kiderlen [13] for $n \geq 3$.

Lemma 4.2. Let $g \in \mathcal{L}$ and let $\mathcal{N}$ be a dense subset of $\mathcal{M}_{o}^{+}\left(S^{n-1}\right)$. Then

$$
\langle g, \mu\rangle \geq 0 \quad \forall \mu \in \mathcal{N}
$$

if and only if there is an $x \in \mathbb{R}^{n}$ such that

$$
g(u)+x \cdot u \geq 0 \quad \forall u \in S^{n-1} .
$$

Proof. Obviously, (4.2) for some $x \in \mathbb{R}^{n}$ implies (4.1). Conversely, assume that (4.1) holds. Since $\mathcal{N}$ is dense in $\mathcal{M}_{o}^{+}\left(S^{n-1}\right)$, (4.1) holds for every measure in $\mathcal{M}_{o}^{+}\left(S^{n-1}\right)$. Let

$$
g=h(L, \cdot)-h(M, \cdot)
$$

with convex bodies $L, M \in \mathcal{K}_{0}^{n}$. Define the inradius of $L$ relative to $M$ by

$$
r(L, M)=\max \left\{\lambda \geq 0: \lambda M \subseteq L+x \text { for some } x \in \mathbb{R}^{n}\right\} .
$$

Choose $x \in \mathbb{R}^{n}$, with $r(L, M) M \subseteq L+x$. By the definition of $r(L, M)$, the contact points of $r(L, M) M$ and $L+x$ are distributed on their boundaries such that

$$
o \in \operatorname{conv}\left\{N(L, y) \cap S^{n-1}: y \in r(L, M) M \cap L+x\right\},
$$

where $N(L, y)$ is the normal cone of $L$ in $y$. Otherwise we could move the body $r(L, M) M$ inside $L+x$ away from the contact points and blow it up, in contradiction to the definition of $r(L, M)$. Let $\mu \in \mathcal{M}_{o}^{+}\left(S^{n-1}\right)$ be concentrated in the set $\left\{N(L, y) \cap S^{n-1}: y \in r(L, M) M \cap L+x\right\}$. By (4.1),

$$
r(L, M)\langle h(M, \cdot), \mu\rangle=\langle h(L, \cdot), \mu\rangle=\langle g+h(M, \cdot), \mu\rangle \geq\langle h(M, \cdot), \mu\rangle .
$$

Thus $r(L, M) \geq 1$, and hence we have for every $u \in S^{n-1}$

$$
g(u)+h(M, u)+x \cdot u=h(L+x, u) \geq r(L, M) h(M, u) \geq h(M, u) .
$$

Using (3.2), and noting that the set of surface area measures of convex bodies is a dense subset of $\mathcal{M}_{o}^{+}\left(S^{n-1}\right)$, we obtain the following geometric consequence of Lemma 4.2 which was proved differently by Weil in 30.

Corollary 4.3. Let $K, L \in \mathcal{K}^{n}$. If $V_{1}(M, K) \leq V_{1}(M, L)$ for every $M \in \mathcal{K}_{0}^{n}$ then there is a vector $x \in \mathbb{R}^{n}$ such that $K+x \subseteq L$.

Note that, if the function $g$ in Lemma 4.2 is zonal, then the vector $x$ in (4.2) can be chosen as a multiple of $\widehat{e}$. The following consequence of Lemma 4.2, which we will use frequently, was also used in the proof of Theorem 3.4, see [13].

Corollary 4.4. Let $\mu \in \mathcal{M}\left(S^{n-1}, \widehat{e}\right)$ and let $\mathcal{N}$ be a dense subset of $\mathcal{M}_{o}^{+}\left(S^{n-1}\right)$. Then

$$
\nu * \mu \in \mathcal{M}_{o}^{+}\left(S^{n-1}\right) \quad \forall \nu \in \mathcal{N}
$$

if and only if $\mu$ is weakly positive. 
Proof. It is clear that (4.3) holds if $\mu$ is weakly positive. Conversely, assume that (4.3) holds. Let $\left(\varphi_{k}\right)_{k \in \mathbb{N}}$ be a zonal approximate identity. Then $\nu * \mu * \varphi_{k} \geq 0$, and by Lemma 2.3. $\mu * \varphi_{k} \in \mathcal{C}^{\infty}\left(S^{n-1}\right)$. Using (2.9), we see that $\left(\nu * \mu * \varphi_{k}\right)(\widehat{e})=$ $\left\langle\mu * \varphi_{k}, \nu\right\rangle \geq 0$ for every $\nu \in \mathcal{N}$. As $\mathcal{C}^{\infty}\left(S^{n-1}\right) \subseteq \mathcal{L}$ (see [28] p.27), by Lemma 4.2 and the remark after Corollary 4.3 , there are $c_{k} \in \mathbb{R}$ such that

$$
\left(\mu * \varphi_{k}\right)(u)+c_{k}(\widehat{e} \cdot u) \geq 0 .
$$

Thus, for nonnegative $f \in \mathcal{C}\left(S^{n-1}\right)$, we have by (2.20)

$$
f * \mu * \varphi_{k} \geq-c_{k} f *(\widehat{e} \cdot .)=-\frac{c_{k}}{n} \pi_{1} f .
$$

By Lemma 2.3, $\mu * \varphi_{k} \rightarrow \mu$ weakly, and thus $f * \mu * \varphi_{k} \rightarrow f * \mu$ uniformly by Lemma 2.1. Hence there exists $b \in \mathbb{R}$ such that $b \geq-c_{k} \pi_{1} f$. Since $\pi_{1} f$ is a linear functional, the sequence $c_{k}$ is bounded. Therefore we can assume that $c_{k} \rightarrow c$.

The main ingredient in the proof of Theorem 1.2 is a classification of translation invariant homogeneous valuations of convex sets. Since the map $K \mapsto S_{n-1}(K, \cdot)$ is a translation invariant valuation (see [28, p.201), we obtain from the definition of Blaschke addition that for all $K, L \in \mathcal{K}_{0}^{n}$ such that $K \cup L \in \mathcal{K}_{0}^{n}$ and $K \cap L \in \mathcal{K}_{0}^{n}$,

$$
(K \cup L) \#(K \cap L)=K \# L \text {. }
$$

Thus, if $\Phi$ is a Blaschke-Minkowski homomorphism, we have by Lemma 4.1 for all $K, L \in \mathcal{K}^{n}$ such that $K \cup L \in \mathcal{K}^{n}$,

$$
\Phi(K \cup L)+\Phi(K \cap L)=\Phi K+\Phi L .
$$

Hence, $\Phi$ is a valuation with respect to Minkowski addition. For further information on valuations of this type see [15] and [16.

The following characterization is due to Hadwiger [11] and McMullen [20]:

Theorem 4.5. A map $\varphi: \mathcal{K}^{n} \rightarrow \mathbb{R}$ is a continuous translation invariant valuation homogeneous of degree $n-1$ if and only if there is a function $g \in \mathcal{C}\left(S^{n-1}\right)$, unique up to addition of a linear function, such that

$$
\varphi(K)=\left\langle g, S_{n-1}(K, \cdot)\right\rangle .
$$

Using Theorem 4.5 and (4.5), we can derive the representation theorem for Blaschke-Minkowski homomorphisms.

Proof of Theorem 1.2, Define a functional $\varphi: \mathcal{K}^{n} \rightarrow \mathbb{R}$ by

$$
\varphi(K)=h(\Phi K, \widehat{e}) \text {. }
$$

Since $S_{n-1}(\lambda K, \cdot)=\lambda^{n-1} S_{n-1}(K, \cdot)$ for $\lambda \geq 0$ and $K \in \mathcal{K}^{n}$, we have by (1.1)

$$
\Phi \lambda K=\lambda^{n-1} \Phi K .
$$

Using (4.6) and (4.5), we see that the map $\varphi$ is a continuous valuation on $\mathcal{K}^{n}$ homogeneous of degree $n-1$. By Theorem 4.5, there is a function $g \in \mathcal{C}\left(S^{n-1}\right)$, unique up to addition of a linear function, such that

$$
\varphi(K)=\left\langle g, S_{n-1}(K, \cdot)\right\rangle .
$$

Since $\varphi$ is invariant under rotations leaving $\widehat{e}$ fixed, the function $g$ is zonal, and thus, by (2.1) and (2.2),

$$
h(\Phi K, \widehat{\eta})=h(\Phi K, \eta \widehat{e})=\left\langle g, S_{n-1}\left(\eta^{-1} K, \cdot\right)\right\rangle=\left\langle\eta g, S_{n-1}(K, \cdot)\right\rangle .
$$


(1.2) now follows from (2.9) and (2.15). To see that $g$ is weakly positive, note that by (3.6), (3.7) and the commutativity of the convolution of zonal functions,

$$
h(\{s(\Phi K)\}, \cdot)=n h(\Phi K, \cdot) *(\widehat{e} \cdot .)=n S_{n-1}(K, \cdot) *(\widehat{e} \cdot .) * g=0 .
$$

Since $s(\Phi K) \in$ relint $\Phi K$ (see 28 , p.43), we have $h(\Phi K, \cdot) \geq 0$. Thus, noting that the set of surface area measures is a dense subset of $\mathcal{M}_{o}^{+}\left(S^{n-1}\right)$, it follows from Corollary 4.4 that $g$ is weakly positive.

For later applications, we state further properties of the generating functions of Blaschke-Minkowski homomorphisms in the following lemma.

Lemma 4.6. Let $g \in \mathcal{C}\left(S^{n-1}, \widehat{e}\right)$ be the generating function of a Blaschke-Minkowski homomorphism.

(a) $g$ is a difference of support functions, i.e. $g \in \mathcal{L}$.

(b) There is a symmetric body of revolution $L \in \mathcal{K}^{n}$, such that

$$
g(u)+g(-u)=h(L, u) \quad \forall u \in S^{n-1} .
$$

Proof. By Lemma 3.8 (a), there are convex bodies $K_{+}, K_{-} \in \mathcal{K}_{0}^{n}$ such that

$$
\delta_{\widehat{e}}-\pi_{1} \delta_{\widehat{e}}=S_{n-1}\left(K_{+}, \cdot\right)-S_{n-1}\left(K_{-}, \cdot\right) .
$$

Since the Dirac measure $\delta_{\widehat{e}}$ is the neutral element for zonal convolution, and as $\left(\pi_{1} \delta_{\widehat{e}}\right)(u)=n \widehat{e} \cdot u$ by (2.20), we obtain

$$
\left(\delta_{\widehat{e}}-\pi_{1} \delta_{\overparen{e}}\right) * g=g-\pi_{1} g=h\left(\Phi K_{+}, \cdot\right)-h\left(\Phi K_{-}, \cdot\right) .
$$

Since $\pi_{1} g$ is a linear functional on $\mathbb{R}^{n}$, there is a vector $x \in \mathbb{R}^{n}$ such that

$$
\left(\pi_{1} g\right)(u)=x \cdot u=h(\{x\}, u) .
$$

Hence $g=h\left(\Phi K_{+}+x, \cdot\right)-h\left(\Phi K_{-}, \cdot\right)$ which proves (a).

To see (b), let $\left\{b_{1}, \ldots, b_{n}\right\}$ be an orthonormal basis in $\mathbb{R}^{n}$ such that $\widehat{e}=b_{n}$. For a vector $x \in \mathbb{R}^{n}$ let $x_{1}, \ldots, x_{n}$ denote its coordinates with respect to $b_{1}, \ldots, b_{n}$. Choose $\beta \in \mathbb{R}^{+}$such that the ellipsoid $E_{\alpha}$ defined by

$$
\frac{x_{1}^{2}+\ldots+x_{n-1}^{2}}{\alpha^{2}}+\frac{x_{n}^{2}}{\beta^{2}} \leq 1
$$

has surface area $S\left(E_{\alpha}\right)=1$. It was shown in 9, p.103, that as $\alpha \rightarrow \infty$, we have $\beta \rightarrow 0$ and

$$
S_{n-1}\left(E_{\alpha}, \cdot\right) \rightarrow \frac{1}{2}\left(\delta_{\widehat{e}}+\delta_{-\widehat{e}}\right)
$$

weakly. By Lemma 2.1.

$$
h\left(\Phi E_{\alpha}, u\right)=\left(S_{n-1}\left(E_{\alpha}, \cdot\right) * g\right)(u) \rightarrow \frac{1}{2}(g(u)+g(-u))
$$

uniformly in $u \in S^{n-1}$. Since $h\left(\Phi E_{\alpha}, \cdot\right)$ converges uniformly, it converges to a support function of a convex body, which proves (b).

An immediate consequence of Lemma 4.6 is the complete classification of all even Blaschke-Minkowski homomorphisms.

Proof of Theorem 1.3. A Blaschke-Minkowski homomorphism is even if and only if its generating function is even. Thus the result follows from Lemma 4.6(b). 
If $g=h(L, \cdot)$ for some body of revolution $L \in \mathcal{K}^{n}$ is the generating function of a Blaschke-Minkowski homomorphism $\Phi$; then by (4.7) and (3.2),

$$
h(\Phi K, \widehat{\eta})=n V_{1}(K, \eta L) .
$$

Since $K_{1} \subseteq K_{2}$ if and only if $h\left(K_{1}, \cdot\right) \leq h\left(K_{2}, \cdot\right)$, the monotonicity of mixed volumes together with (4.8) implies

Corollary 4.7. A Blaschke-Minkowski homomorphism whose generating function is given by $h(L, \cdot)$ for some $L \in \mathcal{K}^{n}$, is monotone with respect to set inclusion.

Note that by Theorem 1.3 and Corollary 4.7, every even Blaschke-Minkowski homomorphism is monotone.

By Lemma 3.1 every map $\Phi: \mathcal{K}^{n} \rightarrow \mathcal{K}^{n}$ of the form

$$
h(\Phi K, \cdot)=S_{n-1}(K, \cdot) * h(L, \cdot)
$$

for some $L \in \mathcal{K}^{n}$ is a Blaschke-Minkowski homomorphism, but in general there are generating functions $g$ of Blaschke-Minkowski homomorphisms that are not support functions. An example of such a map is the (normalized) second mean section operator $M_{2}$ introduced in [7] and further investigated in [12]: Let $\mathcal{E}_{2}^{n}$ be the affine Grassmanian of two-dimensional planes in $\mathbb{R}^{n}$ and $\mu_{2}$ its motion invariant measure, normalized such that $\mu_{2}\left(\left\{E \in \mathcal{E}_{2}^{n}: E \cap B^{n} \neq \varnothing\right\}\right)=\kappa_{n-2}$. Then

$$
h\left(M_{2} K, \cdot\right)=(n-1) \int_{\mathcal{E}_{2}^{n}} h(K \cap E, \cdot) d \mu_{2}(E)-h\left(\left\{z_{n-1}(K)\right\}, \cdot\right)=S_{n-1}(K, \cdot) * g_{2},
$$

where $z_{n-1}(K)$ is the intrinsic $(n-1)$ st moment vector of $K$ (see [28], p.304) and $\Lambda g_{2}$ is given by

$$
\Lambda g_{2}(t)=\arccos (-t) \sqrt{1-t^{2}} .
$$

The function $g_{2}$ is not a support function. Note that the operator $M_{2}$ is not monotone but has the following weak monotonicity property: $M_{2}$ is monotone on those convex bodies having their $(n-1)$ st intrinsic moment vector in the origin. This is similar to the monotonicity property of weakly monotone Minkowski endomorphisms.

We will now give a complete characterization of generating functions of BlaschkeMinkowski homomorphisms in the spirit of a classification result of Weil [32] of generating measures of generalized zonoids. To this end, we need the extension of area measures of convex bodies to the space $\mathcal{L}$ of differences of support functions.

Definition 4.8. Let $g_{i} \in \mathcal{L}, i=1, \ldots, n-1$, with $g_{i}=h\left(K_{i}^{0}, \cdot\right)-h\left(K_{i}^{1}, \cdot\right)$. Then the mixed surface area measure of $g_{1}, \ldots, g_{n-1}$ is defined by

$$
S\left(g_{1}, \ldots, g_{n-1}, \cdot\right)=\sum_{\alpha_{1}, \ldots, \alpha_{n-1} \in\{0,1\}}(-1)^{\alpha_{1}+\ldots+\alpha_{n-1}} S\left(K_{1}^{\alpha_{1}}, \ldots, K_{n-1}^{\alpha_{n-1}}, \cdot\right) .
$$

For a function $f \in \mathcal{C}\left(S^{n-1}\right)$, define

$$
V\left(f, g_{1}, \ldots, g_{n-1}\right)=\left\langle f, S\left(g_{1}, \ldots, g_{n-1}, \cdot\right)\right\rangle .
$$

For $g \in \mathcal{L}$ and $j=1, \ldots, n-1$, the measure $S_{j}(g, \cdot)=S(g, \ldots, g, 1, \ldots, 1, \cdot)$, where $g$ appears $j$ times and 1 appears $n-j-1$ times, is called the area measure of order $j$ of $g$. 
If $\Phi$ is a Blaschke-Minkowski homomorphism, then by Lemma 4.6 (a),

$$
h(\Phi K, \cdot)=S_{n-1}(K, \cdot) * g=S_{n-1}(K, \cdot) * h\left(L_{+}, \cdot\right)-S_{n-1}(K, \cdot) * h\left(L_{-}, \cdot\right),
$$

where $g=h\left(L_{+}, \cdot\right)-h\left(L_{-}, \cdot\right)$. Thus, defining Blaschke-Minkowski homomorphisms $\Phi_{+}$and $\Phi_{-}$with generating functions $h\left(L_{+}, \cdot\right)$ and $h\left(L_{-}, \cdot\right)$ we obtain

$$
h(\Phi K, \cdot)=h\left(\Phi_{+} K, \cdot\right)-h\left(\Phi_{-} K, \cdot\right) .
$$

In the light of (4.9), we need a criterion to determine whether a difference of support functions is in fact a support function. This was established by Weil in [31].

Theorem 4.9. A function $g \in \mathcal{L}$ is the support function of a convex body $K$ if and only if for all $j \in\{1, \ldots, n-1\}$,

$$
S_{j}(g, \cdot) \in \mathcal{M}_{o}^{+}\left(S^{n-1}\right) .
$$

In order to use Theorem 4.9, we need to determine the area measures $S_{j}(\Phi K, \cdot)$. In [9], p.105, the area measures of the convex body with support function $\mu * h(K, \cdot)$, $\mu \in \mathcal{M}^{+}(S O(n))$, were calculated. The result established there extends easily to differences of support functions. Identifying spherical measures with right $S O(n-1)$ invariant measures on $S O(n)$, we get the following lemma.

Lemma 4.10. Let $\Phi$ be a Blaschke-Minkowski homomorphism with generating function $g \in \mathcal{L}$. Then $\left\langle f, S_{j}(\Phi K, \cdot)\right\rangle$ is given by

$$
\int_{\left(S^{n-1}\right)^{j}} V\left(f, \Lambda g\left(u_{1} \cdot\right), \ldots, \Lambda g\left(u_{j} \cdot\right), 1, \ldots, 1\right) d S_{n-1}\left(K, u_{1}\right) \ldots d S_{n-1}\left(K, u_{j}\right) .
$$

Using Lemma 4.10, Theorem 4.9 and the fact that the set of surface area measures of convex bodies forms a dense subset of $\mathcal{M}_{o}^{+}\left(S^{n-1}\right)$, we obtain the following characterization of generating functions of Blaschke-Minkowski homomorphisms.

Theorem 4.11. A function $g \in \mathcal{L}$ is the generating function of a BlaschkeMinkowski homomorphism if and only if, for every $j=1, \ldots, n-1$,

$$
\int_{\left(S^{n-1}\right)^{j}} V\left(f, \Lambda g\left(u_{1} \cdot\right), \ldots, \Lambda g\left(u_{j} \cdot .\right), 1, \ldots, 1\right) d \mu\left(u_{1}\right) \ldots d \mu\left(u_{j}\right) \geq 0
$$

for every nonnegative $f \in \mathcal{C}\left(S^{n-1}\right)$ and every $\mu \in \mathcal{M}_{o}^{+}\left(S^{n-1}\right)$.

\section{ENDOMORPHISMS AND HOMOMORPHISMS OF CONVEX BODIES}

We now turn to the connection between adjoint Minkowski and Blaschke endomorphisms and Blaschke-Minkowski homomorphisms.

Proof of Theorem 1.4. If $\Psi$ and $\Psi^{*}$ are adjoint, then $\Psi$ is weakly monotone and they have the same generating measure $\mu \in \mathcal{M}\left(S^{n-1}, \widehat{e}\right)$. Let $\Phi$ be a BlaschkeMinkowski homomorphism with generating function $g \in \mathcal{C}\left(S^{n-1}, \widehat{e}\right)$. From the commutativity of zonal convolution, it follows that

$$
\begin{aligned}
h\left(\Phi \Psi^{*} K, \cdot\right) & =S_{n-1}\left(\Psi^{*} K, \cdot\right) * g=S_{n-1}(K, \cdot) * \mu * g \\
& =S_{n-1}(K, \cdot) * g * \mu=h(\Phi K, \cdot) * \mu=h(\Psi \Phi K, \cdot) .
\end{aligned}
$$

Thus (a) implies (b) and obviously (b) implies (c).

By the multiplier property, a Blaschke-Minkowski homomorphism $\Phi$ is injective if and only if all the multipliers of $g$ are nonzero. Thus, the multipliers of $\Psi^{*}$ and

$\Psi$ can be determined from $\Phi \circ \Psi^{*}$ and $\Psi \circ \Phi$ and are equal if (1.4) holds. By 
the completeness of the system of spherical harmonics, it follows that (c) implies (a).

Theorem 1.4 shows that the following conjecture is equivalent to Conjecture 3.5

Conjecture 5.1. There exists an injective Blaschke-Minkowski homomorphism whose range is invariant under every Minkowski endomorphism.

In view of this formulation of Conjecture 3.5. we further investigate the range of Blaschke-Minkowski homomorphisms.

Theorem 5.2. The range of every Blaschke-Minkowski homomorphism is nowhere dense in $\mathcal{K}^{n}$.

Proof. We call $K \in \mathcal{K}_{0}^{n}$ Blaschke decomposable if there exist two bodies $K_{1}, K_{2} \in$ $\mathcal{K}_{0}^{n}$ not homothetic to $K$ such that $K=K_{1} \# K_{2}$. By a result of Bronshtein [4], the only Blaschke indecomposable bodies in $\mathcal{K}_{0}^{n}$ are the simplices. Thus, every body in the range of a Blaschke-Minkowski homomorphism with the only possible exception of the image of simplices is decomposable with respect to Minkowski addition.

Since the image of simplices is nowhere dense in $\mathcal{K}^{n}$ and since, on the other hand, the indecomposable bodies with respect to Minkowski addition form a dense subset of $\mathcal{K}^{n}$, the desired result follows.

In the second part of this section we will see that most of the geometric convolution operators we encountered so far do not attain values in the set of polytopes.

Theorem 5.3. Let $\Phi: \mathcal{K}^{n} \rightarrow \mathcal{K}^{n}$ be a Blaschke-Minkowski homomorphism generated by the support function $h(L, \cdot)$ of a body of revolution $L \in \mathcal{K}^{n}$. If there is a convex body $K \in \mathcal{K}_{0}^{n}$ such that $\Phi K$ is a polytope, then there is a constant $c \in \mathbb{R}^{+}$ such that

$$
\Phi=c \Pi \text {. }
$$

Proof. Let $P=\Phi K=\operatorname{conv}\left\{x_{1}, \ldots, x_{k}\right\}$ be a polytope with vertices $x_{1}, \ldots, x_{k}$. Then

$$
h(P, \cdot)=S_{n-1}(K, \cdot) * h(L, \cdot) .
$$

Since the body $L \in \mathcal{K}^{n}$ is unique up to translation, we can assume that $h(L, \cdot) \geq 0$. Let $\mu=\breve{S}_{n-1}(K, \cdot) \in \mathcal{M}^{+}(S O(n))$; then by (1.2)

$$
h(P, \cdot)=\int_{S O(n)} h(\vartheta L, \cdot) d \mu(\vartheta) .
$$

From now on, we consider support functions as positive homogeneous functions on $\mathbb{R}^{n}$. Let $C_{1}, \ldots, C_{k}$ denote the normal cones of the vertices of $P$. Then the support function $h(P, \cdot)$ is linear in every $C_{i}, i=1, \ldots, k$. Thus, by (5.1), we have

$$
\int_{S O(n)} h\left(\vartheta L, v_{1}\right)+h\left(\vartheta L, v_{2}\right)-h\left(\vartheta L, v_{1}+v_{2}\right) d \mu(\vartheta)=0
$$

for all $v_{1}, v_{2} \in C_{i}$. Since support functions are sublinear, the integrand in (5.2) is nonnegative. Thus, as $\mu$ is nonnegative, $h\left(\vartheta L, v_{1}\right)+h\left(\vartheta L, v_{2}\right)=h\left(\vartheta L, v_{1}+v_{2}\right)$ for all $\vartheta$ in the support of $\mu$. For each such $\vartheta$, we thus have

$$
h\left(L, v_{1}\right)+h\left(L, v_{2}\right)=h\left(L, v_{1}+v_{2}\right)
$$

for all $v_{1}, v_{2} \in \vartheta C_{i}$. This implies that $L$ is a polytope itself. But since $L$ is a body of revolution and the only polytopes that are bodies of revolution are the multiples of the segment $[-\widehat{e}, \widehat{e}]$, the desired result follows from (1.3). 
Note that Theorem 1.3 and Theorem 5.3 imply Theorem 1.5 For a corresponding result in dimension two see [27, p.311.

The difference body operator $D: \mathcal{K}^{n} \rightarrow \mathcal{K}^{n}$ is the Minkowski endomorphism defined by

$$
D K=K+(-K) .
$$

The Blaschke body operator $\nabla: \mathcal{K}^{n} \rightarrow \mathcal{K}^{n}$ is the Blaschke endomorphism defined by

$$
\nabla K=K \#(-K)
$$

Corollary 5.4. The only even Blaschke endomorphisms taking values in the set of polytopes are constant multiples of $\nabla$.

If an even Minkowski endomorphism maps a zonoid onto a polytope, then it is a constant multiple of $D$.

Proof. Let $\Psi$ be an even Blaschke endomorphism and let $\Psi K=P$ be a polytope for some $K \in \mathcal{K}_{0}^{n}$. By (1.1), the map $\Pi \circ \Psi$ is an even Blaschke-Minkowski homomorphism such that $\Pi \Psi K$ is a polytope. By Theorem 1.5] and Theorem 1.3, there is a constant $c \in \mathbb{R}^{+}$such that

$$
\Pi \circ \Psi=c \Pi \text {. }
$$

Since $\Pi$ is injective all the even multipliers of $\Pi$ are nonzero. Thus, by (5.3), all even multipliers of $\Psi$ are equal to $c$. Noting that the odd multipliers of even multiplier operators are zero, the result follows.

An analogous argument leads to the second statement.

\section{Geometric inequalities And InduCed operators}

An important open problem in the theory of affine isoperimetric inequalities is the conjectured projection inequality by Petty [24]:

$$
\frac{\kappa_{n}^{n-1}}{\kappa_{n-1}^{n}} V(\Pi K) \geq \kappa_{n} V(K)^{n-1}
$$

with equality if and only if $K$ is an ellipsoid. If (6.1) holds, then, as was shown in [18, it is a strengthened version of the classical isoperimetric inequality $W_{1}(K)^{n} \geq$ $\kappa_{n} V(K)^{n-1}$; compare Lemma 6.7 and (6.6).

In this chapter we will study analogous problems for general Blaschke-Minkowski homomorphisms and related operators which will be introduced in the next theorem. Most of the results in this chapter were established for the projection body operator in 18; see also [17, 19]. The aim of this section is to generalize the results obtained there to general (nontrivial) Blaschke-Minkowski homomorphisms and to show that the crucial tool is a representation of the form of (1.2).

In the following $\Phi: \mathcal{K}^{n} \rightarrow \mathcal{K}^{n}$ shall always denote a nontrivial BlaschkeMinkowski homomorphism.

Theorem 6.1. The map $\Phi$ satisfies the Steiner type formula

$$
\Phi\left(K+\varepsilon B^{n}\right)=\sum_{i=0}^{n-1} \varepsilon^{i}\left(\begin{array}{c}
n-1 \\
i
\end{array}\right) \Phi_{i} K .
$$

The operators $\Phi_{i}: \mathcal{K}^{n} \rightarrow \mathcal{K}^{n}, i=0, \ldots, n-1$, are continuous, translation invariant, rotation intertwining Minkowski valuations and $\Phi_{0}=\Phi$. 
Proof. The desired result is an immediate consequence of Theorem 1.2 and the Steiner formula for the surface area measure of a convex body $K$ (see (3.3)):

$$
S_{n-1}\left(K+\varepsilon B^{n}, \cdot\right)=\sum_{i=0}^{n-1} \varepsilon^{i}\left(\begin{array}{c}
n-1 \\
i
\end{array}\right) S_{n-1-i}(K, \cdot) .
$$

If $g \in \mathcal{C}\left(S^{n-1}, \widehat{e}\right)$ denotes the generating function of $\Phi$, then

$$
h\left(\Phi_{i} K, \cdot\right)=S_{n-1-i}(K, \cdot) * g .
$$

By Minkowski's existence theorem, the area measure $S_{i}(K, \cdot)$ of order $i$ of a convex body $K \in \mathcal{K}_{0}^{n}$ is also the surface area measure $S_{n-1}(L, \cdot)$ of order $n-1$ of some convex body $L \in \mathcal{K}_{0}^{n}$. Thus, the mappings $\Phi_{i}$ are well defined. Since the mappings $K \mapsto S_{i}(K, \cdot)$ are translation invariant valuations, the operators $\Phi_{i}$ are Minkowski valuations.

Note that, by Theorem 1.2 and (3.3), the mappings $\Phi_{i}$ are special cases of more general operators defined on the cartesian product of $n-1$ copies of $\mathcal{K}^{n}$. These mappings are studied in more detail in [29]. In the following we will consider only the operators $\Phi_{i}, i=0, \ldots, n-2$, since $\Phi_{n-1}$ maps every body $K$ to $\Phi B^{n}$ because $S_{0}(K, \cdot)=S_{n-1}\left(B^{n}, \cdot\right)$ is independent of $K$. We remark here that, for $K \in \mathcal{K}_{i}^{n}$, the image $\Phi_{i} K$ is an element of $\mathcal{K}_{0}^{n}$ and $\Phi_{i} L=o$ if $L \in \mathcal{K}_{i+2}^{n} \backslash \mathcal{K}_{i+1}^{n}$.

By (6.2), the $\Phi_{i}$ are multiplier operators, but apart from $\Phi_{0}=\Phi$ and $\Phi_{n-2}$ they cannot be interpreted as additive transformations of convex bodies, since the set of area measures $S_{j}(K, \cdot)$ of order $j$ does not form a cone in $\mathcal{M}_{o}^{+}\left(S^{n-1}\right)$ for $j=2, \ldots, n-2$; see [6].

The operator $\Phi_{n-2}$ is a Minkowski endomorphism. To see this, note that the area measure $S_{1}(K, \cdot)$ of order one is related to the support function $h(K, \cdot)$ by the linear second order differential operator

$$
\Delta_{1}=\Delta_{0}+(n-1),
$$

where $\Delta_{0}$ denotes the Laplace Beltrami operator on $S^{n-1}$; see [9], p.87. We have

$$
\Delta_{1} h(K, \cdot)=S_{1}(K, \cdot)
$$

where this equality is understood in the sense of distributions if $h(K, \cdot)$ is not in $\mathcal{C}^{2}\left(S^{n-1}\right)$. From (6.3), it follows that $S_{1}\left(K_{1}+K_{2}, \cdot\right)=S_{1}\left(K_{1}, \cdot\right)+S_{1}\left(K_{2}, \cdot\right)$, which together with (6.2) shows that $\Phi_{n-2}$ is a Minkowski endomorphism.

As $\Delta_{0}$ is an intertwining operator so is $\Delta_{1}$. Thus, by Theorem 2.8, $\Delta_{1}$ is a multiplier operator. For the following lemma see [9, p.86, and note that multiplier transformations are obviously commutative.

Lemma 6.2. Let $\mu \in \mathcal{M}\left(S^{n-1}, \widehat{e}\right)$ and $\nu \in \mathcal{M}\left(S^{n-1}\right)$. Then

$$
\Delta_{1}(\nu * \mu)=\nu *\left(\Delta_{1} \mu\right)=\left(\Delta_{1} \nu\right) * \mu
$$

in the sense of distributions.

Using Lemma 6.2, we get the following result.

Theorem 6.3. The operator $\Phi_{n-2}$ is a weakly monotone Minkowski endomorphism. 
Proof. We have seen that $\Phi_{n-2}$ is a Minkowski endomorphism. In order to prove that $\Phi_{n-2}$ is weakly monotone, we need to show, by Theorem 3.4 that there is a weakly positive measure $\mu \in \mathcal{M}\left(S^{n-1}, \widehat{e}\right)$ such that $h\left(\Phi_{n-2} K, \cdot\right)=h(K, \cdot) * \mu$.

If $g \in \mathcal{C}\left(S^{n-1}, \widehat{e}\right)$ is the generating function of $\Phi$, then by Lemma 6.2 and (6.3),

$$
h\left(\Phi_{n-2} K, \cdot\right)=S_{1}(K, \cdot) * g=h(K, \cdot) * \Delta_{1} g,
$$

thus we need to show that $\Delta_{1} g$ is a weakly positive measure. Using Lemma 4.6 (a), we have $g=h\left(L_{1}, \cdot\right)-h\left(L_{2}, \cdot\right)$ for some convex bodies $L_{1}, L_{2} \in \mathcal{K}^{n}$. Hence,

$$
\Delta_{1} g=S_{1}\left(L_{1}, \cdot\right)-S_{1}\left(L_{2}, \cdot\right) .
$$

Using again Lemma 6.2 and (6.3), we obtain

$$
S_{1}(\Phi K, \cdot)=S_{n-1}(K, \cdot) * \Delta_{1} g \in \mathcal{M}_{o}^{+}\left(S^{n-1}\right) .
$$

Thus, the desired result follows from Corollary 4.4 and from the fact that the set of surface area measures is a dense subset of $\mathcal{M}_{o}^{+}\left(S^{n-1}\right)$.

For $K, L \in \mathcal{K}^{n}$ and $i=0, \ldots, n-2$, let $W_{i}(K, L)$ denote the mixed volume $V\left(L, K, \ldots, K, B^{n}, \ldots, B^{n}\right)$, where $K$ appears $n-1-i$ times and $B^{n}$ appears $i$ times. Note that, $W_{0}(K, L)=V_{1}(K, L)$. For our further investigations we state the following consequence of Lemma 2.2 .

Lemma 6.4. For $i=0, \ldots, n-2$ and $K, L \in \mathcal{K}^{n}$,

$$
W_{i}\left(K, \Phi_{i} L\right)=W_{i}\left(L, \Phi_{i} K\right) .
$$

Proof. Let $g \in \mathcal{C}\left(S^{n-1}, \widehat{e}\right)$ denote the generating function of $\Phi$. From (3.4), the definition of $W_{i}(K, L)$ and Lemma 2.2 it follows that

$$
\begin{aligned}
W_{i}\left(K, \Phi_{i} L\right) & =\frac{1}{n}\left\langle h\left(\Phi_{i} L, \cdot\right), S_{n-1-i}(K, \cdot)\right\rangle=\frac{1}{n}\left\langle S_{n-1-i}(L, \cdot) * g, S_{n-1-i}(K, \cdot)\right\rangle \\
& =\frac{1}{n}\left\langle S_{n-1-i}(L, \cdot), S_{n-1-i}(K, \cdot) * g\right\rangle=W_{i}\left(L, \Phi_{i} K\right) .
\end{aligned}
$$

The Shephard problem asks whether for $K, L \in \mathcal{K}_{0}^{n}$,

$$
\operatorname{vol}_{n-1}\left(K \mid u^{\perp}\right)=h(\Pi K, u) \leq h(\Pi L, u)=\operatorname{vol}_{n-1}\left(L \mid u^{\perp}\right)
$$

for every $u \in S^{n-1}$ implies $V(K) \leq V(L)$. Obviously, (6.4) is equivalent to $\Pi K \subseteq$ $\Pi L$. As was shown independently by Petty [23] and Schneider [25], the answer to Shephard's problem in general is no, but if the body $L$ is a zonoid, the answer is yes. The crucial tool in the proof of the latter statement is a special case of Lemma 6.4. In fact, an analogous result can be shown for general Blaschke-Minkowski homomorphisms.

Corollary 6.5. Let $K \in \mathcal{K}_{i}^{n}$ and $L \in \Phi_{i} \mathcal{K}_{i}^{n}$. Then, for $i=0, \ldots, n-2$,

$$
\Phi_{i} K \subseteq \Phi_{i} L \quad \Rightarrow \quad W_{i}(K) \leq W_{i}(L)
$$

and $W_{i}(K)=W_{i}(L)$ only if $K$ and $L$ are translates.

Proof. From the monotonicity of mixed volumes, Lemma 6.4 and the fact that $L=\Phi_{i} L_{0}$ for some convex body $L_{0} \in \mathcal{K}_{i}^{n}$, it follows that

$$
W_{i}\left(K, \Phi_{i} L_{0}\right)=W_{i}\left(L_{0}, \Phi_{i} K\right) \leq W_{i}\left(L_{0}, \Phi_{i} L\right)=W_{i}\left(L, \Phi_{i} L_{0}\right)=W_{i}(L) .
$$

Using the generalized Minkowski inequality

$$
W_{i}(K, L)^{n-i} \geq W_{i}(K)^{n-1-i} W_{i}(L),
$$


with equality if and only if $K$ and $L$ are homothetic, we thus get

$$
W_{i}(K) \leq W_{i}(L),
$$

with equality only if $K$ and $L$ are homothetic. But homothetic bodies of equal $i$ th quermassintegral must be translates of each other.

The special case $i=0, \Phi=\Pi$ of Corollary 6.5 is the result of Schneider and Petty. The following result is a generalization of Corollary [3.9, which follows from (6.2).

Corollary 6.6. For $i=0, \ldots, n-2$ and $K \in \mathcal{K}^{n}$,

$$
W_{n-1}\left(\Phi_{i} K\right)=r_{\Phi} W_{i+1}(K)
$$

where $r_{\Phi} \in \mathbb{R}^{+}$is the radius of the ball $\Phi B^{n}$.

We will now prove an upper bound for the $i$ th quermassintegral of $\Phi_{i} K$.

Theorem 6.7. For $i=0, \ldots, n-2$ and $K \in \mathcal{K}^{n}$,

$$
W_{i+1}(K)^{n-i} \geq \frac{\kappa_{n}^{n-1-i}}{r_{\Phi}^{n-i}} W_{i}\left(\Phi_{i} K\right)
$$

where $r_{\Phi} \in \mathbb{R}^{+}$is the radius of the ball $\Phi B^{n}$. There is equality only if $\Phi_{i} K$ is a ball.

Proof. Let $K \in \mathcal{K}^{n}$ and $0 \leq i \leq n-2$. From inequality (3.1), we get by repeated application, the inequality

$$
W_{n-1}(K)^{n-i} \geq \kappa_{n}^{n-1-i} W_{i}(K),
$$

where, for $K \in \mathcal{K}_{n-1}^{n}$, there is equality if and only if $K$ is a ball. Taking $K=\Phi_{i} K$ and using Corollary 6.6. gives the desired result.

In the following we will investigate, for $K \in \mathcal{K}_{i}^{n}$, the similarity invariant ratio

$$
\psi_{i}(K)=\frac{W_{i}\left(\Phi_{i} K\right)}{W_{i}(K)^{n-1-i}} .
$$

By a standard technique, using Blaschke's selection theorem, it can be shown that $\psi_{i}$ attains a minimum on $\mathcal{K}_{i}^{n}$. From the next theorem it follows that the extremal bodies $K$ of this minimum have the property that $K$ and $\Phi_{i}^{2} K$ are homothetic.

Theorem 6.8. If $K \in \mathcal{K}_{i}^{n}$ and $0 \leq i \leq n-2$, then

$$
\psi_{i}(K) \geq \psi_{i}\left(\Phi_{i} K\right)
$$

with equality if and only if $K$ and $\Phi_{i}^{2} K$ are homothetic.

Proof. Let $K, L \in \mathcal{K}_{i}^{n}$. From the generalized Minkowski inequality (6.5) together with Lemma 6.4, we obtain

$$
W_{i}\left(L, \Phi_{i} K\right)^{n-i}=W_{i}\left(K, \Phi_{i} L\right)^{n-i} \geq W_{i}(K)^{n-1-i} W_{i}\left(\Phi_{i} L\right),
$$

with equality if and only if $K$ and $\Phi_{i} L$ are homothetic. Setting $L=\Phi_{i} K$, gives

$$
W_{i}\left(\Phi_{i} K\right)^{n-i} \geq W_{i}(K)^{n-1-i} W_{i}\left(\Phi_{i}^{2} K\right),
$$

with equality if and only if $K$ and $\Phi_{i}^{2} K$ are homothetic. 
In the case $i=n-2$, there is a result of Kiderlen [13 that $K$ and $\Psi^{2} K$, for a nontrivial weakly monotone Minkowski endomorphism $\Psi$, are homothetic if and only if $K$ is a ball, where the combinations of the identity map and the reflection in the origin are the trivial Minkowski endomorphisms. Thus, Theorem 6.7 and Theorem 6.8 together with Kiderlen's result imply Theorem 1.6 .

In the proof of Theorem 1.6, we have used only that $\Phi_{n-2}$ is a weakly monotone (nontrivial) Minkowski endomorphism. In fact, inequality (1.5) with equality cases is valid for every nontrivial weakly monotone Minkowski endomorphism; compare also [26], p.70, for a related result. The reason why we chose the more restrictive formulation of Theorem 1.6 is the author's belief that Petty's conjectured projection inequality holds in a more general form for every Blaschke-Minkowski homomorphism and its induced operators

$$
W_{i+1}(K)^{n-i} \geq \frac{\kappa_{n}^{n-1-i}}{r_{\Phi}^{n-i}} W_{i}\left(\Phi_{i} K\right) \geq \kappa_{n} W_{i}(K)^{n-1-i},
$$

giving a family of strengthened versions of the classical inequalities (3.1) between consecutive quermassintegrals. The inequalities (3.1) are special cases of (6.6) for the Blaschke-Minkowski homomorphisms $K \mapsto c B\left(W_{1}(K), o\right)$, where $c \in \mathbb{R}^{+}$and $B\left(W_{1}(K), o\right)$ is the ball with center in the origin and radius $W_{1}(K)$. It is possible to show that the case $i=0$ of (6.6) implies the inequality for all other values of $i$; for a proof compare the argument for the projection body operator in [18, p.57.

\section{ACKNOWLEDGEMENTS}

The work of the author was supported by the Austrian Science Fund (FWF), within the scope of the project "Affinely associated bodies", Project Number: P16547-N12 and the project "Phenomena in high dimensions" of the European Community, Contract Number: MRTN-CT-2004-511953. For their helpful remarks the author is obliged to Monika Ludwig and Rolf Schneider.

\section{REFERENCES}

1. C. Berg, Corps convexes et potentiels sphériques, Danske Vid. Selsk. Mat.-Fys. Medd. 37, 6 (1969), 1-64. MR0254789(40:7996)

2. J. Bourgain and J. Lindenstrauss, Projection bodies, Geometric aspects of functional analysis (1986/87), Springer, Berlin (1988), 250-270. MR950986 (89g:46024)

3. E.D. Bolker, A class of convex bodies, Trans. Amer. Math. Soc. 145 (1969), 323-345. MR 0256265(41:921)

4. E.M. Bronshtein, Extremal H-convex bodies (in Russian), Sibirskii Mat. Zh. 20 (1979), 412415. English Translation: Siberian Math. J. 20, 295-297. MR 530507 (80f:52002)

5. R.J. Gardner, Geometric Tomography, Cambridge University Press, 1995. MR.1356221 (96j:52006)

6. P. Goodey, R. Schneider, On the intermediate area functions of convex bodies, Math. Z. 173 (1980), 185-194. MR.583385 (81k:52010)

7. P. Goodey and W. Weil, The determination of convex bodies from the mean of random sections, Math. Proc. Camb. Phil. Soc. 112 (1992), 419-430. MR1171176 (93i:52008)

8. P. Goodey and W. Weil, Zonoids and generalizations, Handbook of Convex Geometry (P.M. Gruber and J.M. Wills, eds.), North-Holland, Amsterdam, 1993, 1297-1326. MR:1243010 (95g:52015)

9. E. Grinberg and G. Zhang, Convolutions, transforms, and convex bodies, Proc. London Math. Soc. (3) $\mathbf{7 8}$ (1999), 77-115. MR1658156 (99m:52009)

10. H. Groemer, Geometric Applications of Fourier Series and Spherical Harmonics, Cambridge University Press, 1996. MR:1412143 (97j:52001) 
11. H. Hadwiger, Vorlesungen über Inhalt, Oberfläche und Isoperimetrie, Springer, Berlin, 1957. MR 0102775 (21:1561)

12. D. Hug and R. Schneider, Stability results involving surface area measures of convex bodies, Rendiconti Del Circolo Matematica Di Palermo 70 (2002), 21-51. MR.1962583 (2004b:52004)

13. M. Kiderlen, Blaschke- and Minkowski-Endomorphisms of convex bodies, Trans. Amer. Math. Soc., to appear. MR2238926

14. D.A. Klain and G.C.Rota, Introduction to geometric probability, Cambridge University Press, Cambridge, 1997. MR 1608265 (2001f:52009)

15. M. Ludwig, Projection bodies and valuations, Adv. Math. 172 (2002), 158-168. MR.1942402 (2003j:52012)

16. M. Ludwig, Minkowski valuations, Trans. Amer. Math. Soc. 357 (2005), no. 10, 4191-4213. MR 2159706 (2006f:52005)

17. E. Lutwak, Mixed projection inequalities, Trans. Amer. Math. Soc. 287 (1985), no. 1, 91-105. MR766208 (86c:52015)

18. E. Lutwak, On quermassintegrals of mixed projection bodies, Geom. Dedicata 33 (1990), 5158. MR.1042624 (91b:52008)

19. E. Lutwak, Inequalities for mixed projection bodies, Trans. Amer. Math. Soc. 339 (1993), no. 2, 901-916. MR.1124171 (93m:52011)

20. P. McMullen, Continuous translation invariant valuations on the space of compact convex sets, Arch. Math. 34, 377-384. MR593954 (81m:52013)

21. P. McMullen, Valuations and dissections, Handbook of Convex Geometry, Vol. B (P.M. Gruber and J.M. Wills, eds.), North Holland, Amsterdam, 1993, 933-990. MR1243000 (95f:52018)

22. P. McMullen and R. Schneider, Valuations on convex bodies, Convexity and its applications (P.M. Gruber and J.M. Wills, eds.), Birkhäuser, 1983, 170-247. MR731112 (85e:52001)

23. C.M. Petty, Projection bodies, Proceedings, Coll. Convexity, Copenhagen, 1965, Kobenhavns Univ. Mat. Inst. (1967), 234-241. MR0216369 (35:7203)

24. C.M. Petty, Isoperimetric problems, Proc. Conf. on Convexity and Combinatorial Geometry, Univ. of Oklahoma, June 1971 (1972), 26-41. MR0362057|(50:14499)

25. R. Schneider, Zu einem Problem von Shephard über die Projektionen konvexer Körper, Math. Z. 101 (1967), 71-82. MR0218976 (36:2059)

26. R. Schneider, Equivariant endomorphisms of the space of convex bodies, Trans. Amer. Math. Soc. 194 (1974), 53-78. MR0353147 (50:5633)

27. R. Schneider, Bewegungsäquivariante, additive und stetige Transformationen konvexer Bereiche, Arch. Math. 25 (1974), 303-312. MR0344999 (49:9738)

28. R. Schneider, Convex Bodies: The Brunn-Minkowski Theory, Cambridge University Press, 1993. MR 1216521 (94d:52007)

29. F.E. Schuster, Volume Inequalities and Additive Maps of Convex Bodies, in preparation.

30. W. Weil, Decomposition of convex bodies, Mathematika 21 (1974), 19-25. MR0365359 $(51: 1611)$

31. W. Weil, Über den Vektorraum der Differenzen von Stützfunktionen konvexer Körper, Math. Nachr. 59 (1974), 353-369. MR0341283 (49:6033)

32. W. Weil, Kontinuierliche Linearkombination von Strecken, Math. Z. 148 (1976), 71-84. MR 0400052 (53:3887)

33. W. Weil, On surface area measures of convex bodies, Geom. Dedicata 9 (1980), 299-306. MR:585937 (81m:52014)

Institut für Diskrete Mathematik and Geometrie, Technische Universität Wien, Wiedner Hauptstrasse 8-10/1046, 1040 Wien, Austria

E-mail address: fschuster@osiris.tuwien.ac.at 